\title{
The FEWS Index: Fixed Effects with a Window Splice
}

\author{
Frances Krsinich ${ }^{1}$
}

\begin{abstract}
This article describes the estimation of quality-adjusted price indexes from 'big data' such as scanner and online data when there is no available information on product characteristics for explicit quality adjustment using hedonic regression. The longitudinal information can be exploited to implicitly quality-adjust the price indexes. The fixed-effects (or 'time-product dummy') index is shown to be equivalent to a fully interacted time-dummy hedonic index based on all price-determining characteristics of the products, despite those characteristics not being observed. In production, this can be combined with a modified approach to splicing that incorporates the price movement across the full estimation window to reflect new products with one period's lag without requiring revision. Empirical results for this fixed-effects window-splice (FEWS) index are presented for different data sources: three years of New Zealand consumer electronics scanner data from market-research company GfK; six years of United States supermarket scanner data from market-research company IRI; and 15 months of New Zealand consumer electronics daily online data from MIT's Billion Prices Project.
\end{abstract}

Key words: Big data; scanner data; online data; hedonic regression; quality adjustment.

\section{Introduction}

Ensuring that consumer price indexes reflect only price change is important, and is traditionally achieved by pricing a fixed basket of goods over time. However, products such as consumer electronics are rapidly evolving, and have correspondingly shorter life cycles in the market. This makes the traditional approach of pricing a representative fixed basket challenging.

The potential for using so-called 'big data' such as scanner data or online data to measure price change with higher accuracy and frequency makes the challenge of rapid product change for price measurement increasingly pertinent. Price indexes in the context of official statistics need to use the data that are available, and ideally their estimation should be automatable.

Price measurement using scanner data has been an active area of research for Statistics New Zealand over the last five years. Collaborative research with Statistics Netherlands

1 Statistics New Zealand - Prices Unit, PO Box 2922 Wellington 6041, New Zealand. Email: frances.krsinich@stats.govt.nz

Acknowledgments: The author would like to thank Chris Pike, manager of the Prices Unit at Statistics New Zealand, for his much-appreciated support and encouragement of this research; Richard Arnold, Alistair Gray, Jan de Haan and three anonymous referees for helpful discussion and comments; and Alberto Cavallo for sharing online data from the Billion Prices Project.

Disclaimer: The opinions, findings, recommendations, and conclusions expressed in this article are those of the author. They do not necessarily represent those of Statistics NZ, which takes no responsibility for any omissions or errors in the information contained here. 
(de Haan and Krsinich 2014) on a new method called the imputation Törnqvist RYGEKS (ITRYGEKS) has established it as benchmark index which appropriately quality adjusts, both for the changing quality mix of products being bought and for the implicit price movements of new products entering, and old products disappearing, from the market.

Supermarket scanner data and online data, however, do not contain sufficient information on characteristics to use methods such as the ITRYGEKS or the multilateral time-dummy (TD) hedonic index. This has led to a revisiting of the fixed-effects index (also known as the time-product dummy (TPD) index) that was used to benchmark the New Zealand housing rentals index (Krsinich 2011b). 'Fixed effects' refers to the fitting of product-specific identifiers. This can be seen as the model controlling for the bundles of characteristics corresponding to each product, rather than controlling directly for the characteristics.

This article demonstrates, theoretically and empirically, that the fixed-effects index is equivalent to a fully interacted time-dummy hedonic index based on all characteristics that are constant across time at the barcode, or detailed product-specification, level. Note that this does not advocate the explicit use of fully interacted hedonic models in practice, rather it demonstrates what is implicitly achieved by the fixed-effects model.

It is also shown empirically that this fully interacted time-dummy hedonic index is virtually the same as the more common main-effects time-dummy hedonic index when a comprehensive set of price-determining characteristics are available for inclusion in the main-effects hedonic model.

Because the fixed-effects index requires at least two price observations before a new product is nontrivially incorporated into the estimation, a modified approach to the 'splicing' or updating of the price indexes in production is required. This window-splice approach uses the movement across the entire estimation window, rather than just the movement of the most recent period, thereby incorporating a catch-up revision factor reflecting the implicit price movements associated with the introduction of new products in the period after their introduction along with an updating of the predicted fixed effects for all products observed. The combination of a fixed-effects index estimation with the window splicing is referred to as the 'fixed-effects window-splice' (FEWS) index.

So, if there is longitudinal price and quantity information at a detailed productspecification level, the FEWS approach can be used to produce nonrevisable qualityadjusted price indexes.

The article is structured as follows:

Section 2 gives the background to the development of the FEWS index - from benchmarking the New Zealand housing rentals index to evaluating this approach in the context of both supermarket scanner data and online data, and then incorporating fixedeffects indexes for mobile phones and televisions in the New Zealand import price index from the December 2013 quarter.

In Section 3, a result from de Haan and Hendriks (2013) for the time-dummy hedonic index is restated in terms of categorical characteristics. This is then extended to include interaction terms, showing that the fixed-effects index is algebraically equivalent to a fully interacted time-dummy hedonic index that explicitly incorporates all characteristics of the products.

In Section 4, a simulation is used to give some intuition for the equivalence of the fully interacted time-dummy hedonic index and the fixed-effects indexes by basing the product 
identifiers used in the fixed-effects index on the same cut-down set of characteristics included in the time-dummy hedonic index.

Section 5 describes how the window-splice incorporates a catch-up revision factor along with the most recent period's movement to maintain the integrity of the long-term index, in particular by revising for the implicit price movements of new products entering the market in the period after their introduction.

In Section 6, empirical results for the FEWS index are compared to other indexes for a range of data sources:

- New Zealand monthly aggregated consumer electronics scanner data from marketresearch company GfK, with quantities and comprehensive sets of characteristic information for each product category.

- United States weekly aggregated supermarket scanner data from IRI marketing, with quantities sold but no characteristic information. For manageability, the data is aggregated to a monthly level for the analysis.

- New Zealand daily consumer electronics web-scraped online data from the Billion Prices Project, with no characteristics or quantities (Billion Prices Project, 2016).

Section 7 concludes the article.

\section{Background}

A fixed-effects approach was used by Statistics New Zealand to retrospectively benchmark the performance of the current matched-sample approach to measuring the price movement of housing rentals. Due to the lack of sufficient characteristics in the housing rental survey data, the longitudinal nature of the data was exploited by fitting a fixed-effects model - that is, the fitting of dwelling-specific intercepts - to implicitly control for all time-invariant characteristics of the surveyed rental dwellings. As pointed out by de Haan (2015a), this is conceptually very similar to the quality-adjustment approach of 'overlap pricing', though it is based on the price movements of all matched products, rather than just one product being replaced.

This approach was controversial at the time, and so Krsinich (2011b) extended a result from Aizcorbe et al. (2003) to show that the implicit price movement being estimated for new rental dwellings by the retrospective fixed-effects index was appropriate.

De Haan and Krsinich (2014) presented and empirically tested the imputation Törnqvist rolling year GEKS (ITRYGEKS) index. This is an extension of the rolling year GEKS (RYGEKS) of Ivancic et al. (2011).

The GEKS index takes the geometric average of all bilateral superlative indexes (e.g., Törnqvist or Fisher indexes) within an estimation window. A rolling window is used with the GEKS index to make it nonrevisable for production, with the latest month's movement spliced onto the previous index number each month. This results in the rolling year GEKS (RYGEKS) index. The window length is usually just over one year, that is, 13 months for a monthly index.

In contrast to the RYGEKS, the ITRYGEKS is based on bilateral time-dummy hedonic indexes rather than superlative indexes. This enables the ITRYGEKS index to reflect the implicit price movements of new and disappearing products on entry and exit, which the RYGEKS does not do. 
It was noted as an interesting result in de Haan and Krsinich (2014) that the rolling year time-product dummy (RYTPD) index - that is, the fixed-effects index - tended to sit closer to the benchmark ITRYGEKS than did the RYGEKS, despite utilising only product identifiers rather than explicitly incorporating information on characteristics.

Krsinich (2013) modified the fixed-effects index by splicing on the movement across the entire window rather than just the movement of the most recent period. This further improved the performance of the fixed-effects index, as measured against the benchmark ITRYGEKS.

Also in Krsinich (2013), it was first noted that the time-product dummy index appeared to be equivalent to a fully interacted time-dummy hedonic model and, therefore, might in a way be more comprehensively quality-adjusting than the ITRYGEKS, which is based on main-effects time-dummy hedonic bilateral indexes.

De Haan and Hendriks (2013) explored the use of fixed-effects indexes for producing high-frequency price indexes from online data, deriving expressions for both the timedummy hedonic and fixed-effects indexes. However, implausible empirical results for women's t-shirts, along with their observation that "measuring quality-adjusted price indexes without information on item characteristics is just not possible", led the authors to conclude that the fixed-effects index is not appropriate for products "where quality change is important".

One hypothesis about this apparent bias in the women's t-shirts index is that, because fashion is highly seasonal, there was probably an almost complete replacement of old products with new products between seasons. The only matched products on which to base the fixed-effects estimation would be products undergoing heavy discounting. See Appendix 2 for the intuition behind this. This suggests that a condition for using the fixedeffects index might be that there is at least some minimum percentage of products that are matched, and not undergoing seasonal discounting, between any two successive periods. Alternatively, the regression assumption of constant coefficients within the estimation window may be violated for these very seasonal products.

In the December 2013 quarter, Statistics NZ introduced fixed-effects indexes into the production of the New Zealand import price index for both mobile phones and televisions. For the major brands of these two products, comprehensive data on import prices and quantities is available longitudinally at a detailed product level. Because the previous quarter of the New Zealand import price index is revisable, the window-splicing aspect of the FEWS index is not required for the incorporation of new products' price movements into the index, though it may be incorporated into production in the future to improve the estimation.

\section{Theory}

In this section, results from de Haan and Hendriks (2013) are extended to show that the fixed-effects index is equivalent to a fully interacted time-dummy hedonic index where all characteristics that define products are treated as categorical. "Fully interacted" means that the regression equation includes the interaction terms for all possible combinations of main effects.

In the case of scanner data, where barcodes change with any change in a pricedetermining characteristic, this means the fixed-effects index (with barcodes, or their 
equivalent, as the product identifiers) is equivalent to a fully interacted time-dummy hedonic index where all price-determining characteristics are explicitly included in the hedonic model.

\subsection{The Main-Effects Time-Dummy Hedonic Index}

The estimating equation for the (unweighted) time-dummy hedonic index can be stated as:

$$
\ln p_{i}^{t}=\delta^{0}+\sum_{t=1}^{T} \delta^{t} D_{i}^{t}+\sum_{k=1}^{K} \beta_{k} z_{i k}+\varepsilon_{i}^{t}
$$

where $p_{i}^{t}$ is the price of product $i$ in period $t ; D_{i}^{t}$ is a dummy variable with the value 1 for period $t$ and 0 otherwise; $z_{i k}$ is the quantity of characteristic $k$ and $\varepsilon_{i}^{t}$ is the error term.

The time-dummy hedonic index is then derived from the estimated parameters on time as follows:

$$
P_{T D}^{0, t}=\exp \left(\hat{\delta}^{t}\right)
$$

Since Equation (1) includes only main effects for the $z$ characteristics, we make this explicit by referring to its corresponding index as the 'main-effects time-dummy' hedonic index $P_{T D(M E)}$.

From Equation (3) in de Haan and Hendriks (2013) we obtain:

$$
P_{T D(M E)}^{0 t}=\exp \left(\hat{\delta}^{t}\right)=\frac{\prod_{i \in S^{t}}\left(p_{i}^{t}\right)^{\frac{1}{N^{t}}}}{\prod_{i \in S^{0}}\left(p_{i}^{0}\right)^{\frac{1}{N^{0}}}} \exp \left[\sum_{k=1}^{K} \hat{\beta}_{k}\left(\bar{z}_{k}^{0}-\bar{z}_{k}^{t}\right)\right]
$$

where $S^{0}$ and $S^{t}$ are the set of products available in times 0 and $t$ respectively and $N^{t}$ is the number of items in time $t$.

They also extend the formulation to the weighted case. For simplicity, this theory section is restricted to the unweighted case, but the simulation in Section 4 shows the equivalence of the two indexes in the weighted case.

The exponential factor is the quality-adjustment factor, which adjusts the ratio of geometric mean prices for any changes in the average characteristics of products between period $t$ and the base period 0 .

Any change in a characteristic in scanner data will correspond to a change in barcode. In online data, a change in characteristic will correspond to a changed product identifier, or model name. So the set of values that characteristics can take is discrete, as a consequence of the set of barcodes or product identifiers being discrete.

This means that when estimating price indexes from scanner or online data, all characteristics can be treated as categorical. Characteristics correspond to a discrete set of product specifications. That is, even numeric characteristics such as 'screen size' for computers or 'number of pixels' for cameras will take a discrete set of values across the set of product specifications.

A convenient feature of treating all characteristics as categorical is that no parametric form is imposed on the hedonic models.

Note also that, rather than building predictive models, these models are conditioning price-determining characteristics out of the parameters estimated for time, from which the price index is derived. The parameters estimated on the characteristics other than time are 
of no interest in themselves. Nevertheless the parameter estimates may be useful as regression diagnostics.

With all the characteristics being modelled as categorical, (3) can be restated as follows.

$$
P_{T D(M E)}^{0 t}=\exp \left(\hat{\delta}^{t}\right)=\frac{\prod_{i \in S^{t}}\left(p_{i}^{t}\right)^{\frac{1}{N^{t}}}}{\prod_{i \in S^{0}}\left(p_{i}^{0}\right)^{\frac{1}{N^{0}}}} \exp \left[\sum_{l=1}^{L} \hat{\beta}_{l}\left(\bar{D}_{l}^{0}-\bar{D}_{l}^{t}\right)\right]
$$

where $L$ is the total number of categories across the $k$ characteristics (minus a base category for each of the $k$ characteristics) and $\bar{D}_{l}^{t}$ is the proportion of $i \in S^{t}$ with the characteristic $D_{l}$.

\subsection{The Fully Interacted Time-Dummy Hedonic Index}

Following the same reasoning as in de Haan and Hendriks (2013), an equivalent expression to (4) can be derived for the fully interacted time-dummy hedonic index, that is, the index derived from a hedonic model that includes the main effects and all the interactions between characteristics. For $n$ characteristics this would be the main effects, the two-way interactions, the three-way interactions, and so on up to the $n$-way interactions.

For simplicity, and without loss of generality, products with just two characteristics are considered here. For example, characteristics $A$ and $B$, each of which has three categories 1,2 , and 3 . The four main effects correspond to the dummy variables for each of $A 1, A 2$, $A 3, B 1, B 2$, and $B 3$ (less the two base categories for each of characteristics $A$ and $B$ ). The eight second-order interactions are the dummy variables for each of $A 1 B 1, A 1 B 2, \ldots$, A3B3 (less one base category).

The estimating equation for the full hedonic model can be written:

$$
\ln p_{i}^{t}=\delta^{0}+\sum_{t=1}^{T} \delta^{t} D_{i}^{t}+\sum_{l=1}^{L} \beta_{l} D_{i l}+\sum_{m=1}^{M} \beta_{m} D_{i m}+\varepsilon_{i}^{t}
$$

where $p_{i}^{t}$ is the price of item $i$ in period $t ; D_{i l}$ are the dummy variables for the $L$ main effects and $D_{i m}$ are the dummy variables for the $M$ second-order interactions, with $\beta_{l}$ and $\beta_{m}$ the corresponding parameters; $\delta^{0}$ is the intercept; $\delta^{t}$ are the time-dummy parameters (from which the index is derived); and $\varepsilon_{i}^{t}$ are the random errors.

Appendix 1 follows the approach of de Haan and Hendriks (2013) to give the full derivation from (5) of the fully interacted time-dummy hedonic index shown in Equation (6).

$$
\begin{aligned}
P_{T D(f u l l)}^{0 t} & =\exp \left(\hat{\delta}^{t}\right) \\
& =\frac{\prod_{i \in S^{t}}\left(p_{i}^{t}\right)^{\frac{1}{N^{t}}}}{\prod_{i \in S^{0}}\left(p_{i}^{0}\right)^{\frac{1}{N^{0}}}} \exp \left[\sum_{l=1}^{L} \hat{\beta}_{l}\left(\bar{D}_{l}^{0}-\bar{D}_{l}^{t}\right)+\sum_{m=1}^{M} \hat{\beta}_{m}\left(\bar{D}_{m}^{0}-\bar{D}_{m}^{t}\right)\right]
\end{aligned}
$$

This is the time-dummy hedonic index with quality adjustment for the change in characteristics in terms of not only the main effects, but all the interactions of characteristics. So the quality adjustment is more comprehensive than that of the maineffects time-dummy index of equation (4). 


\subsection{The Fixed-Effects Index}

Similarly to the case of the time-dummy hedonic index, de Haan and Hendriks (2013) show that, with an estimating equation for the (unweighted) fixed-effects index of

$$
\ln p_{i}^{t}=\alpha+\sum_{t=1}^{T} \delta^{t} D_{i}^{t}+\sum_{i=1}^{N-1} \gamma_{i} D_{i}+\varepsilon_{i}^{t}
$$

where $D_{i}$ is a dummy variable that has the value of 1 if the observation relates to item $i$ and 0 otherwise, the fixed-effects index can be formulated as follows:

$$
P_{F E}^{0 t}=\exp \left(\hat{\delta}^{t}\right)=\frac{\prod_{i \in S^{t}}\left(p_{i}^{t}\right)^{\frac{1}{N^{t}}}}{\prod_{i \in S^{0}}\left(p_{i}^{0}\right)^{\frac{1}{N^{0}}}} \exp \left[\overline{\hat{\gamma}}^{0}-\overline{\hat{\gamma}}^{t}\right]
$$

where $\overline{\hat{\gamma}}^{0}=\sum_{i \in S^{0}} \hat{\gamma}_{i} / N^{0}$ and $\overline{\hat{\gamma}}^{t}=\sum_{i \in S^{t}} \hat{\gamma}_{i} / N^{t}$ are the sample means of the estimated fixed effects.

The exponential terms in (6) and (8) are the same, because the fixed effect for any item $i$ is, by definition, the net effect of the parameters corresponding to the 'bundle' of characteristics belonging to $i$, where the characteristics are constant across time at the item, or product, level. That is, the fixed effect is the sum of the parameters on the main effects and all the interactions for the characteristics of the product.

To illustrate using the example given in Subsection 3.2, consider an item $i$ with the characteristics $A 3$ and $B 1$ in time $t$.

In the fully interacted time-dummy hedonic index formulation of Equation (6), this item will contribute $\hat{\beta}_{A 3}+\hat{\beta}_{B 1}+\hat{\beta}_{A 3 B 1}$ to the expression in the brackets which, when exponentiated, is the quality-adjustment factor.

In the fixed-effects index formulation of Equation (8), the item contributes a fixed effect of $\hat{\gamma}_{i}$ to the corresponding bracketed term.

Since, by definition, the fixed effect is the net effect of the characteristics of the item, $\hat{\gamma}_{i}=\hat{\beta}_{A 3}+\hat{\beta}_{B 1}+\hat{\beta}_{A 3 B 1}$, and it follows that Equation (6) = Equation (8).

So, the fixed-effects index is equivalent to the fully interacted time-dummy hedonic index where all price-determining, and therefore product-identifying, characteristics are included in the time-dummy hedonic index.

Price indexes should appropriately reflect the relative expenditures on different products. The following section demonstrates the equivalence of the fixed-effects and fully interacted time-dummy hedonic indexes empirically for the weighted case (using expenditure shares as weights) by using a subset of three characteristics to both include in the fully interacted time-dummy hedonic index and define the product identifiers used in the fixed-effects index.

\section{Simulation}

Scanner data from market-research company GfK is used for digital cameras for the three years from July 2008 to June 2011.

To run a fully interacted time-dummy hedonic model, just three of the approximately 40 available characteristics for digital cameras are incorporated into the model. 
These characteristics are brand (with 22 categories), depth of device in millimetres (295 categories), and photos per second (78 categories).

To estimate the corresponding fixed-effects index, product identifiers are defined based on these three characteristics.

For example, a product of brand $A$, with a depth of device in millimetres of twelve, and photos per second of 100 , could be assigned an identifier of 'A_12_100'. A product of brand $B$, depth of device in millimetres of 25 , and photos per second of 200 , could be called 'B_25_200'. Note that these product identifiers are text strings, and therefore convey no information about the corresponding characteristics to the estimation process.

Figure 1 shows the resulting indexes. The fully interacted time-dummy hedonic index (TD fully interacted) and the fixed-effects index (FE) are precisely the same, despite no characteristics being explicitly incorporated into the fixed-effects estimation.

We also show the standard time-dummy hedonic index, which includes only the main effects for the three included characteristics. The difference between this main-effects time-dummy hedonic index and the fully interacted time-dummy hedonic index shows there is extra price-determining information in the interactions between these three characteristics that is not reflected in the parameters on the main effects. It is likely that particular combinations of characteristics correspond to particular models of camera, and therefore the interactions are indirectly reflecting the price-effects of other characteristics related to those models.

Note that these indexes are estimated on the full set of data - that is, they are not the rolling-window versions which would be used in production to produce nonrevisable indexes, as discussed below in Section 5.

As the set of characteristics included in the main-effects time-dummy hedonic index increases, the difference between the main-effects and fully interacted time-dummy hedonic indexes decreases. This is shown in Subsection 6.1 by the very close correspondence between the time-dummy movement-splice (TDMS) index and the fixedeffects movement-splice (FEMS) indexes for eight consumer electronics products.

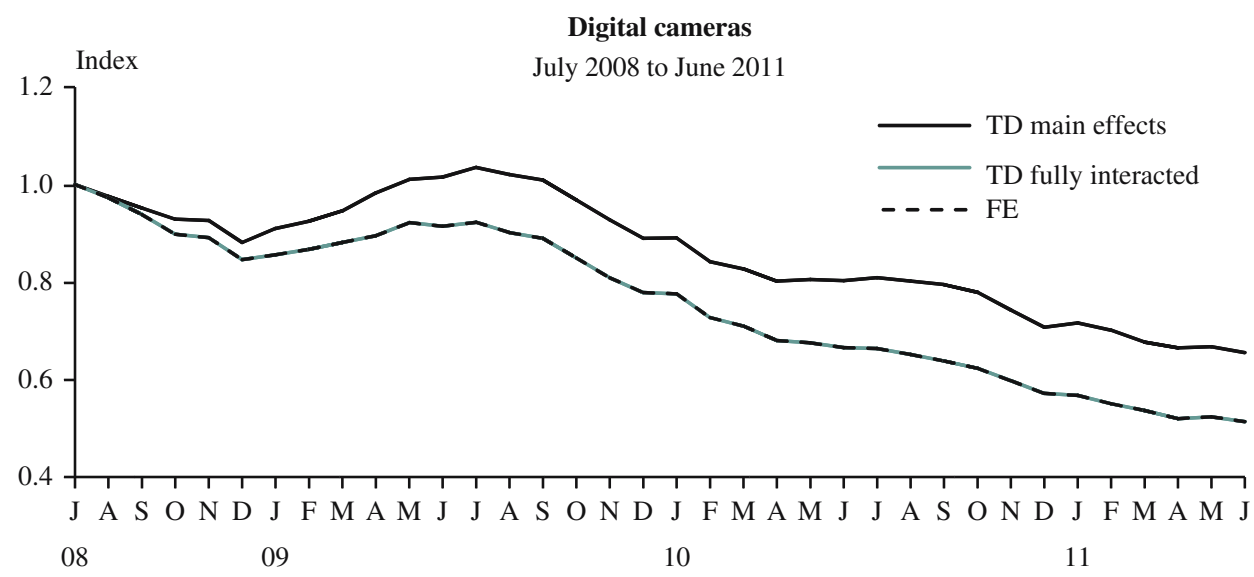

Source: GfK

Fig. 1. Comparison of fully interacted time-dummy hedonic and fixed-effects indexes 


\section{The Window Splice}

Consumer price indexes need to be nonrevisable. This is usually achieved by incorporating a 'rolling window' for the set of data used to estimate the index, and splicing on the most recent period's movement to the previously published index number.

Krsinich (2013) proposed a modified approach to the splicing that uses the movement across the entire estimation window rather than just the most recent period's movement. This 'window splice' is a simplified version of a suggestion for improving the splicing of the RYGEKS made by Melser (2011).

Appendix 2 gives the intuition for why the fixed-effects model needs at least two price observations to include a new product nontrivially in the estimation. It also shows how the fixed-effects estimates are improved with more price observations for each product.

The window splice enables a form of implicit revision, which maintains the integrity of the index over the longer term, not only incorporating the implicit price movements of new products being introduced, but also enabling the updating of the fixed-effects estimates as more prices are observed for each product.

Consider a situation where the index over a seven-quarter period is based on three successive five-quarter estimation windows. We use a quarterly, rather than monthly, index to simplify the example.

In practice, when estimating time-dummy hedonic indexes, the index will generally change only slightly for previous periods with each subsequent window's estimations. For the illustration purpose, we here show an extreme situation of quite significant change to the index with each subsequent estimation window.

In the example shown in Figure 2, the index estimated from the first-quarter window of data has a constant increase of ten percent per quarter, the index estimated on window two is steeper, with a 15 percent per quarter increase, and the third window's index reflects a 20 percent increase in each quarter.

The standard approach of splicing on just the most recent periods - the 'movement splice' - would result in the index showing a ten percent increase in each of the first five quarters from Y1Q1 to Y2Q1, then 15 percent from Y2Q1 to Y2Q2, and then 20 percent from Y2Q2 to Y2Q3. This is shown in Figure 3.

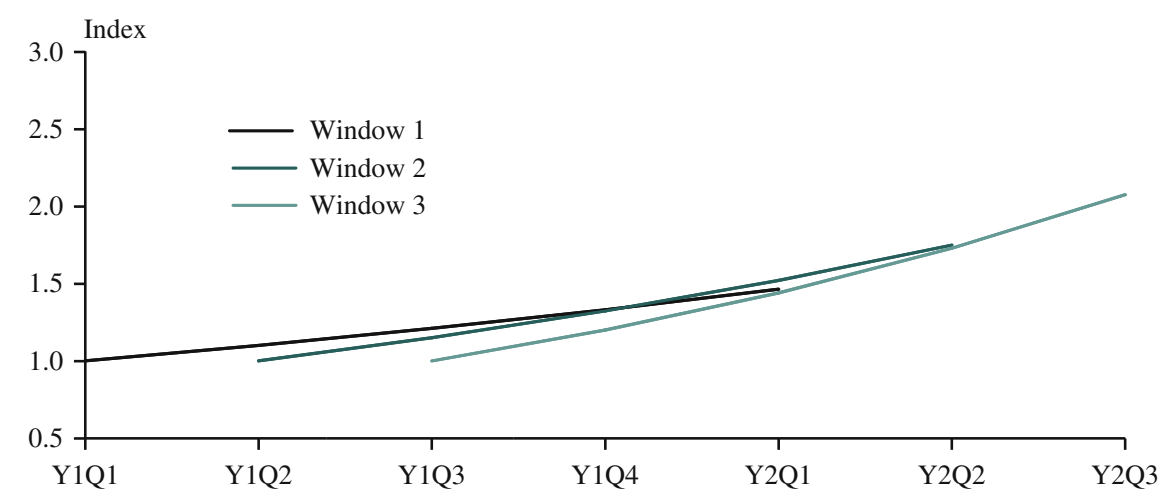

Fig. 2. Indexes estimated from three successive five-quarter windows 


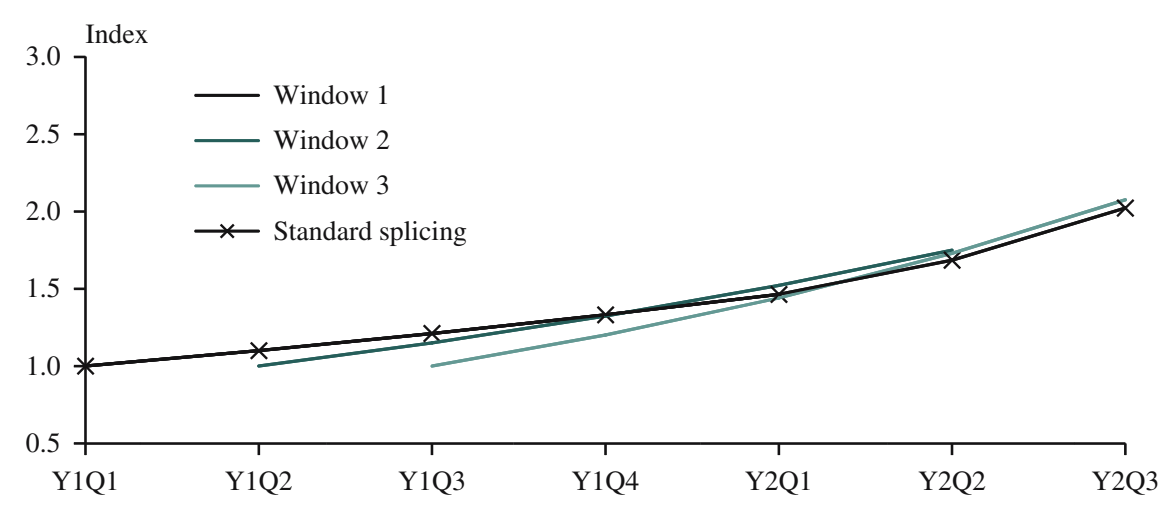

Fig. 3. Splicing on the most recent period's movement

Extending the notation introduced by de Haan (2015b), we can express the movementspliced index for period $T+t$ (where the estimation window is of length $T+1$ and $t>0$ ) as follows:

$$
P_{M S}^{0, T+t}=P_{[0, T]}^{0, T} \times \frac{P_{[1, T+1]}^{1, T+1}}{P_{[1, T+1]}^{1, T}} \times \frac{P_{[2, T+2]}^{2, T+2}}{P_{[2, T+2]}^{2, T+1}} \times \ldots \times \frac{P_{[t, T+t]}^{t, T+t}}{P_{[t, T+t]}^{t, T+t-1}}
$$

where $P_{[c . d]}^{a, b}$ is the index from period $a$ to period $b$, estimated using data from period $c$ to period $d$.

So, for the seven-quarter example shown above in Figure 3, the index for the last period Y2Q3 will be:

$$
P_{M S}^{0,6}=P_{[0,4]}^{0,4} \times \frac{P_{[1,5]}^{1,5}}{P_{[1,5]}^{1,4}} \times \frac{P_{[2,6]}^{2,6}}{P_{[2,6]}^{2,5}}
$$

The problem with this approach is that the revised movement for previous periods is not incorporated into the longer-term index movement. This could result in a bias, likely to accumulate over time, which we can refer to as 'splice drift'.

Another approach to the splicing would be to incorporate the movement across the entire estimation window, so that the longer-term index always reflects the most up-to-date estimation of not only the most recent period's movement, but the entire estimation window.

If we were able to revise the index, then this splicing of the most recent window's index each time would be as shown in Figure 4. The revised index shows a ten percent increase from Y1Q1 to Y1Q2, a 15 percent increase from Y1Q2 to Y1Q3, and 20 percent quarterly increases between Y1Q3 and Y2Q3. So, at all points the index is based on the most recent estimation window available.

We can formulate this 'revisable-index splicing' as follows:

$$
P_{R S}^{0, T+t}=P_{[0, T]}^{0,1} \times P_{[1, T+1]}^{1,2} \times \ldots \times P_{[t, T+t]}^{t, T+t}
$$

which, for the last period Y2Q3 in the example shown in Figure 4, will be

$$
P_{R S}^{0,6}=P_{[0,4]}^{0,1} \times P_{[1,5]}^{1,2} \times P_{[2,6]}^{2,6}
$$




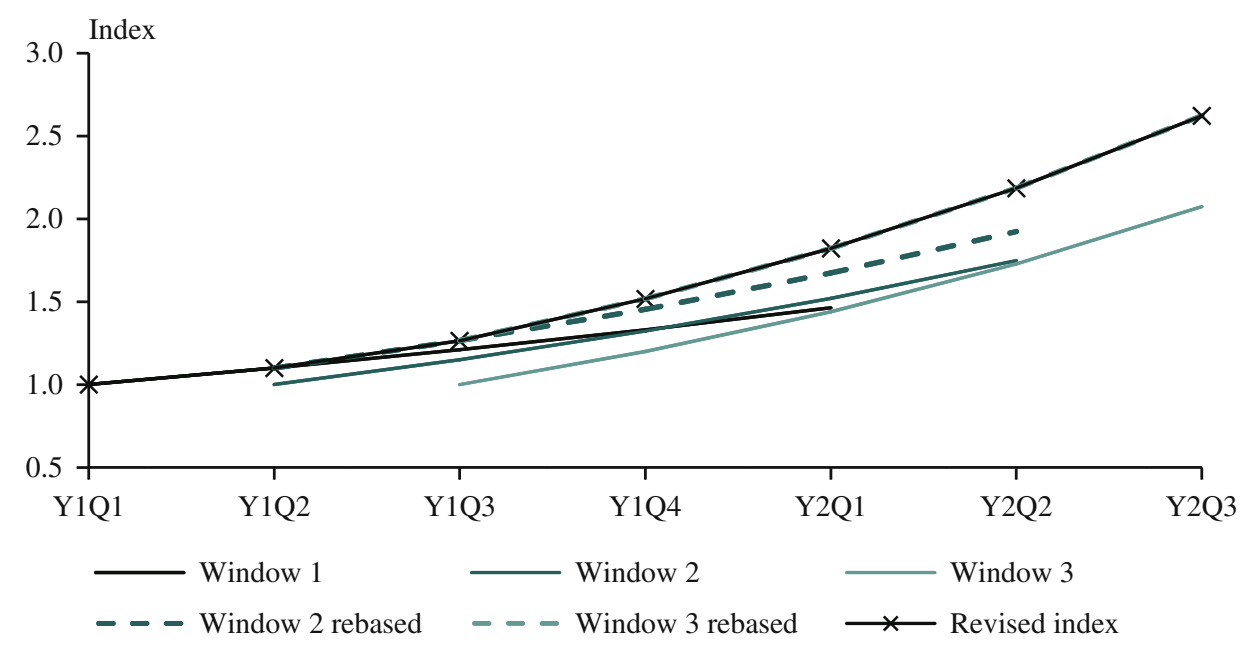

Fig. 4. Revising the index with the most recent window's estimation

And in this quarter, the previous quarter's index can be revised from

$$
P_{R S}^{0,5}=P_{[0,4]}^{0,1} \times P_{[1,5]}^{1,5}
$$

to

$$
P_{R S(r e v 1)}^{0,5}=P_{[0,4]}^{0,1} \times P_{[1,5]}^{1,2} \times P_{[2,6]}^{2,5}
$$

However, consumer price indexes are generally unrevisable.

Instead, a 'catch-up' factor can be incorporated into the most recent period's movement, to maintain the index at the level it would be if revision had been possible. This is the approach taken with the 'window-splice' of the FEWS index, illustrated in Figure 5.

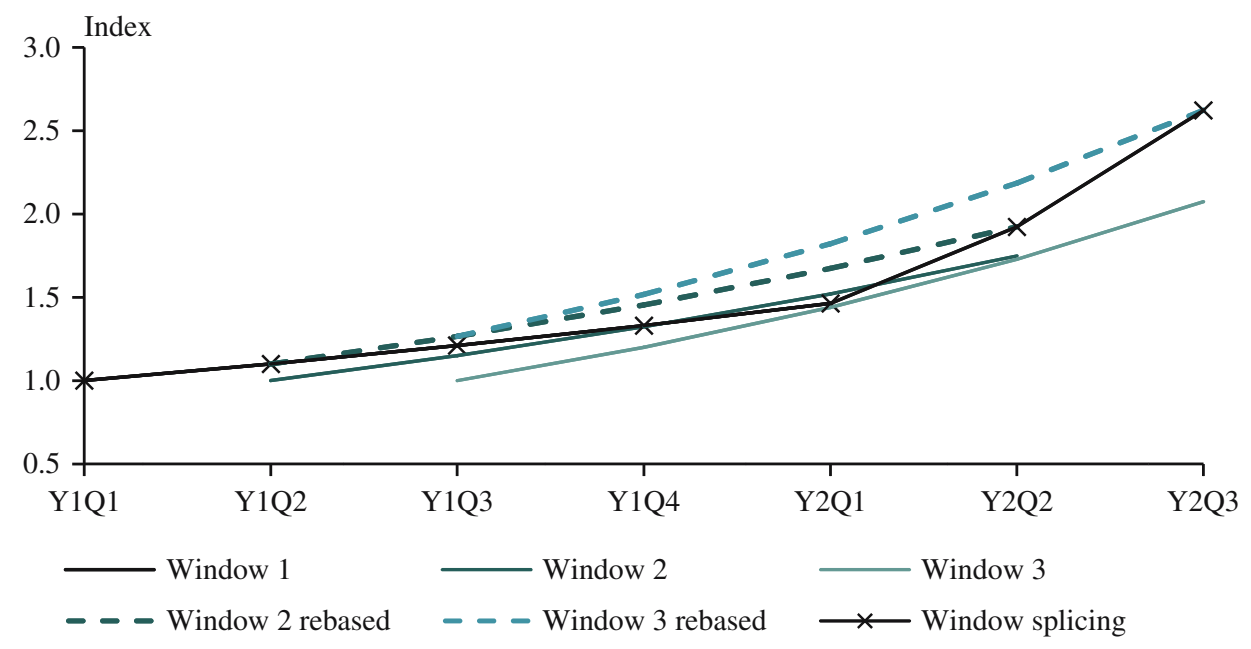

Fig. 5. Incorporating a window splice when the index cannot be revised 
As for the revisable-index splicing, the window splicing for period $\mathrm{T}+\mathrm{t}$ (where $\mathrm{t}>0$ ) can be expressed as:

$$
P_{W S}^{0, T+t}=P_{[0, T]}^{0,1} \times P_{[1, T+1]}^{1,2} \times \ldots \times P_{[t, T+t]}^{t, T+t}
$$

And so the index for the last period $\mathrm{Y} 2 \mathrm{Q} 2$ in the example is similarly

$$
P_{W S}^{0,6}=P_{[0,4]}^{0,1} \times P_{[1,5]}^{1,2} \times P_{[2,6]}^{2,6}
$$

That is, the direct forms of the revisable-spliced and window-spliced indexes are the same.

There is a trade-off with the window-splice approach - between the quality of the most recent period's estimated movement and that of the longer-term index. However, in the case of fixed effects where at least two price observations are required before a new product contributes nontrivially to the estimation, the window splice ensures there is no systematic bias due to the continual omission of the implicit price movements of new products entering the index. It does this by incorporating a revision for them in the period after their introduction. In addition, this window splicing also incorporates implicit revisions for the improvement of the fixed-effects estimates with more price observations.

So the published, and unrevisable, quarter's movement incorporates a revision factor that adjusts the index to the level it would be if revision were possible. Obviously, with such an artificial example as this, where the estimated index changes significantly with each successive window's estimation, the effect on the most recent period's movement is quite substantial. But in practice the change to previous period's movements with the addition of one period of data is usually very small, so there would not be such a sacrifice in the quality of the most recent period's movement to maintain an unbiased longer-term index.

The FEWS index combines this window splice with the fixed-effects index to produce price indexes that are both quality-adjusted and nonrevisable.

To reiterate - the implicit price movement from $t-1$ to $t$ of a new product at time $t$ will not be reflected at time $t$ until the window splice is incorporated for the index estimated at time $t+1$. That is, the implicit price movements will be reflected in the appropriate period, but with one period's lag.

De Haan (2015b) compares movement splicing with window splicing. From his Equation (12) we can state the FEWS index for period $T+1$ as:

$$
P_{F E W S}^{0, T+1}=\frac{P_{F E[1, T+1]}^{1, T}}{P_{F E[0, T]}^{1, T}} \times P_{F E M S}^{0, T+1}
$$

where $P_{F E M S}^{0, T+1}$ is the fixed-effects movement-splice index for period $T+1$.

The ratio of $P_{F E[1, T+1]}^{1, T}$ and $P_{F E[0, T]}^{1, T}$ is the implicit revision factor and de Haan's Equation (13) shows it can be expressed as

$$
\frac{P_{F E[1, T+1]}^{1, T}}{P_{F E[0, T]}^{1, T}}=\exp \left[\sum_{i \in S^{1}} s_{i}^{1}\left(\hat{\gamma}_{i[1, T+1]}-\hat{\gamma}_{i[0, T]}\right)-\sum_{i \in S^{T}} s_{i}^{T}\left(\hat{\gamma}_{i[1, T+1]}-\hat{\gamma}_{i[0, T]}\right)\right]
$$


where:

$\hat{\gamma}_{i[t, T+t]}$ is the predicted fixed effect for product $i$ estimated on the window from $t$ to $T+t$

$s_{i}^{t}$ is the expenditure share of product $i$ in period $t$, and $S^{t}$ is the set of products observed in period $t$.

\section{Empirical Results}

Empirical results for the FEWS index are shown, on a range of data sources with different features:

- Scanner data from market-research company GfK. Three years of monthly average prices with a full set of characteristics, and their associated quantities, for eight consumer electronics products in New Zealand.

- Scanner data from market-research company IRI. Six years of weekly average prices for 30 supermarket products in the United States. Information on quantities sold is available in the data, but there is little information on the characteristics of products.

- Online data from the Billion Prices Project at MIT. Fifteen months of daily webscraped online data for four consumer electronics products in New Zealand. This data has little information on characteristics of products, and no quantity information.

Descriptive statistics on the three data sources are shown in Appendix 3, showing the average number of distinct products per month (for GfK and IRI scanner data) and per week (for the BPP online data), along with the match rates after the first year for each product category, to indicate the levels of product turnover. Table 1 shows the variables available in each of the three data sources, and the price indexes that could be compared for each data source, given these varying data limitations.

Table 1. Price indexes compared for different data sources

\begin{tabular}{llll}
\hline & Available variables & \multicolumn{2}{c}{ Price indexes compared } \\
\cline { 3 - 4 } Data source & Traditional & Hedonic-based \\
\hline $\begin{array}{l}\text { Scanner data (GfK) } \\
\text { consumer electronics } \\
\text { products }\end{array}$ & $\begin{array}{c}\text { Prices, quantities, } \\
\text { characteristics }\end{array}$ & $\begin{array}{c}\text { chained } \\
\text { Törnqvist }\end{array}$ & TDMS, FEMS, FEWS \\
$\begin{array}{l}\text { Scanner data (IRI) } \\
\text { supermarket products }\end{array}$ & Prices, quantities & $\begin{array}{c}\text { chained } \\
\text { Törnqvist }\end{array}$ & FEMS, FEWS \\
$\begin{array}{l}\text { Online data (PriceStats) } \\
\text { consumer electronics } \\
\text { products }\end{array}$ & Prices & $\begin{array}{c}\text { chained } \\
\text { Jevons }\end{array}$ & $\begin{array}{c}\text { unweighted FEMS, } \\
\text { unweighted FEWS }\end{array}$ \\
\hline
\end{tabular}

\subsection{Scanner Data with both Quantities and Characteristics: Eight New Zealand Consumer Electronics Products}

The FEWS index is estimated on three years of monthly average prices from New Zealand scanner data for eight consumer electronics products, from market-research company GfK. This data has extensive information on characteristics (around 40 different characteristics for each product) with corresponding total prices and quantities. From 


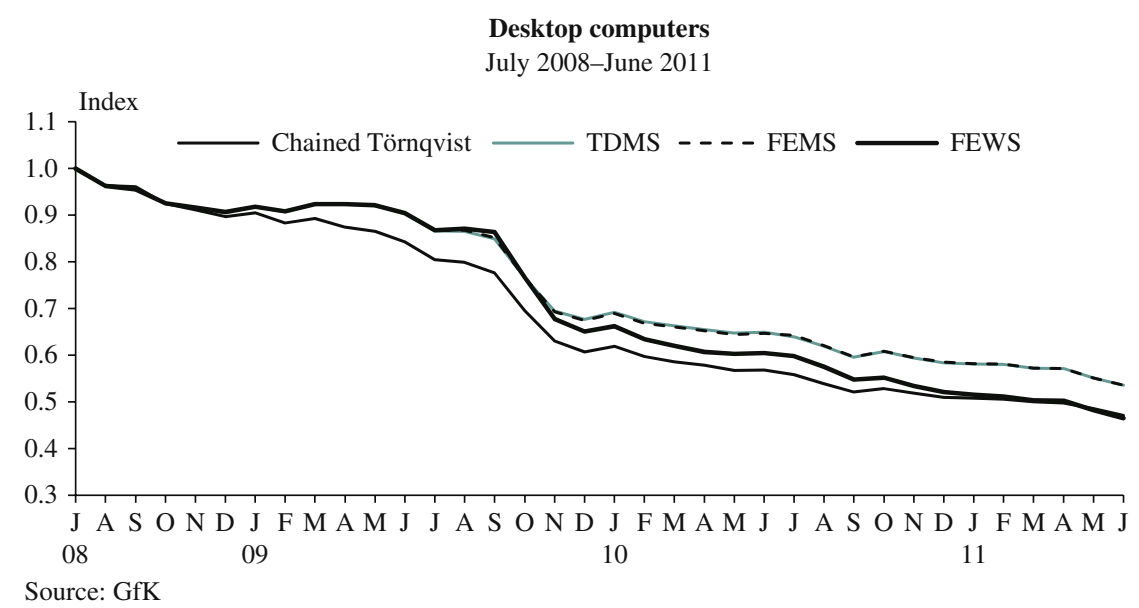

Fig. 6. Comparison of FEWS to other indexes for desktop computers

these, average monthly prices and expenditures for each combination of characteristics is derived. There is no outlet or retailer information available in the data, so this is not controlled for.

Because there is a full set of characteristics and expenditure information in the data, the FEWS index can be compared with the time-dummy movement-splice (TDMS) hedonic index, which explicitly incorporates the characteristics into a multilateral hedonic model. The TDMS is more commonly known as a 'rolling-year time-dummy' (RYTD) index, but we are renaming it in this article to make the splicing method explicit, for the comparison with the other indexes.

The fixed-effects movement-splice (FEMS) index is shown, in order to see the impact of the window splicing and the (implicit) addition of interaction terms separately.

The more traditional chained-Törnqvist index is also included in the comparison:

$$
P_{T}^{t-1, t}=\prod_{i \in M}\left(\frac{p_{i t}}{p_{i t-1}}\right)^{\sigma_{i}}
$$

Digital cameras

July 2008-June 2011

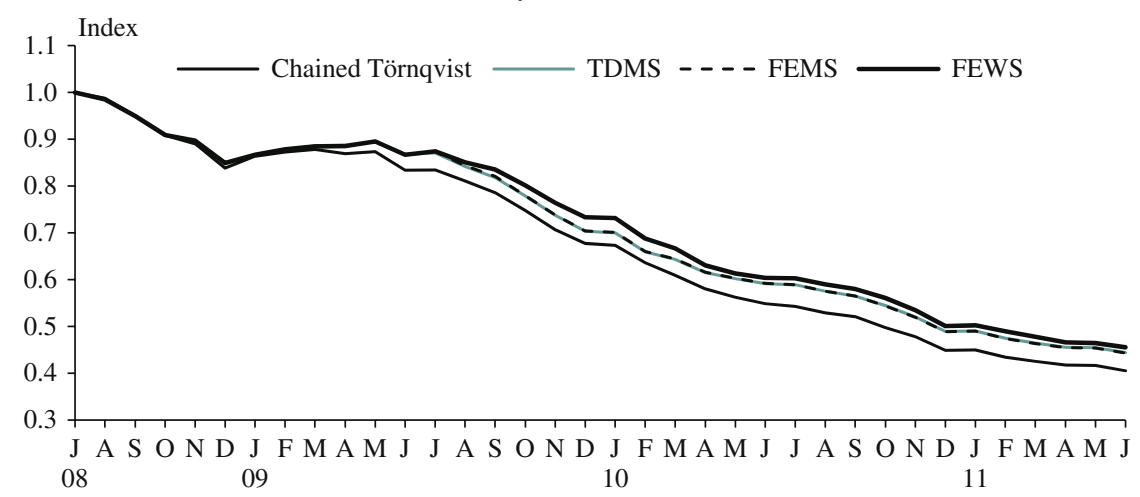

Source: GfK

Fig. 7. Comparison of FEWS to other indexes for digital cameras 


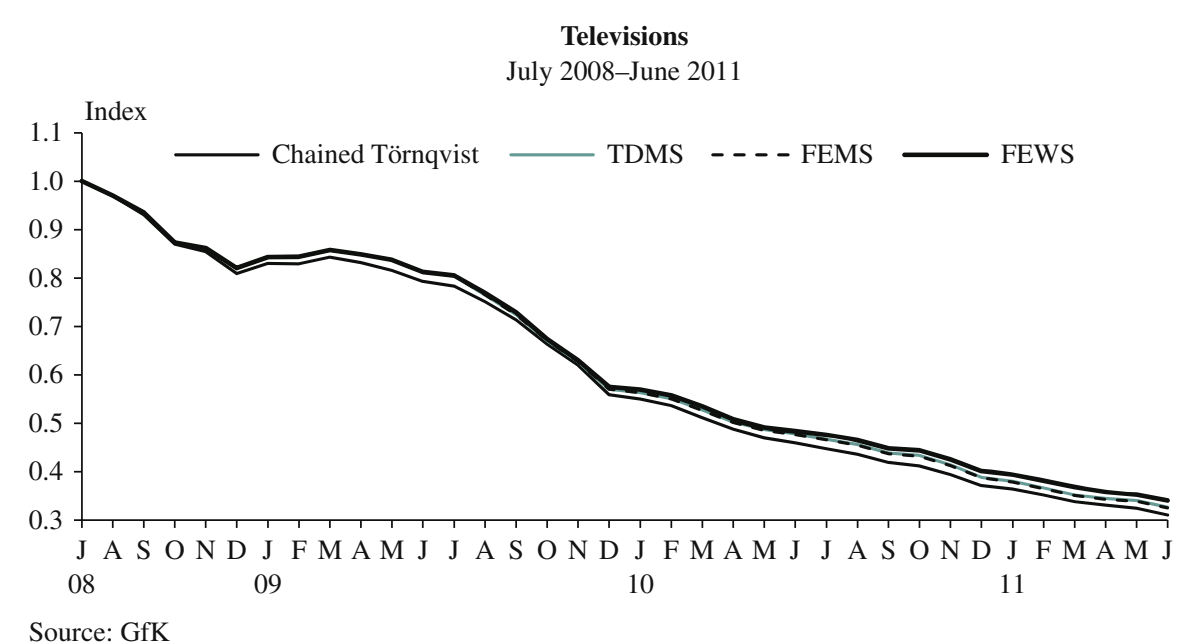

Fig. 8. Comparison of FEWS to other indexes for televisions

where $M$ is the set of products existing in both periods and $\sigma_{i}$ is the arithmetic average of the share of expenditure on product $i$ in periods $t-1$ and $t$ :

$$
\sigma_{i}=\frac{S_{i}^{t}+S_{i}^{t-1}}{2}
$$

Note that while earlier versions of this article included the RYGEKS index in the comparison, recent work by Lamboray and Krsinich (2015) has demonstrated that the GEKS index is biased by product turnover, and has suggested a modification called the 'intersection GEKS' (intGEKS). The issue of whether the RYGEKS, as the nonrevisable counterpart of the standard GEKS, is appropriate in situations of high product turnover, such as consumer electronics, is therefore currently an open question, and so it is excluded from these results.

Figures 6 to 8 show the four indexes for desktop computers, digital cameras and televisions, respectively. Table 2 shows the value of the indexes at the end of the threeyear study period for all eight consumer electronics product categories analysed.

For all three product categories graphed, the TDMS and FEMS indexes are virtually identical, suggesting that the implicit inclusion of interaction terms in the FEWS index adds little extra price-determining information for these product categories.

Table 2. Value of indexes at June 2011 (base $=1$ at July 2008)

\begin{tabular}{lcccc}
\hline & Chained Törnqvist & TDMS & FEMS & FEWS \\
\hline Camcorders & 0.392 & 0.383 & 0.382 & 0.425 \\
Desktop computers & 0.471 & 0.536 & 0.536 & 0.465 \\
Digital cameras & 0.406 & 0.445 & 0.444 & 0.456 \\
DVD players and recorders & 0.588 & 0.595 & 0.633 & 0.631 \\
Laptop computers & 0.434 & 0.460 & 0.452 & 0.437 \\
Microwaves & 0.825 & 0.928 & 0.920 & 0.919 \\
Portable media players & 0.721 & 0.741 & 0.723 & 0.705 \\
Televisions & 0.311 & 0.327 & 0.325 & 0.340 \\
\hline
\end{tabular}


Interestingly, the incorporation of the window splice for desktop computers results in a more rapidly declining index from late 2009, which could correspond to the implicit price movements associated with the introduction of new desktop computers being lower than those of existing desktop computers starting around then.

For both digital cameras and televisions, the FEWS index declines slightly less rapidly than the FEMS and TDMS, while the chained Törnqvist declines more rapidly.

Table 2 shows the very close correspondence of the TDMS and the FEMS indexes for most of the products.

\subsection{Scanner Data with Quantities but no Characteristics: 30 United States Supermarket Products}

Six years of weekly aggregated supermarket scanner data from IRI marketing (see Bronnenberg et al. 2008) is used to compare the FEWS index with the FEMS and the chained-Törnqvist indexes. The data is aggregated to a monthly level for the sake of manageability.

Unlike the consumer electronics scanner data from GfK, supermarket scanner data tends to contain few, if any, characteristics with which to run either the ITRYGEKS or the timedummy hedonic (TD) methods, which both rely on hedonic models that explicitly incorporate a full set of price-determining characteristics. However, outlet identifiers are available, and these are incorporated into the product identifiers to reflect and control for the different levels of service that can be experienced across outlets, and which can therefore be considered as part of the overall 'product'.

We compare the FEWS index with the FEMS index and the (monthly) chainedTörnqvist index. For most of the product categories, the three indexes track each other relatively closely. In general, there does not appear to be significant chain drift in the chained-Törnqvist index, in contrast to the findings in earlier research such as de Haan and van der Grient (2011) and Ivancic et al. (2011).

For brevity, then, graphs for just four of the 30 supermarket product categories analysed are shown in figures 9 to 12 - deodorant, milk, razors, and facial tissues. These

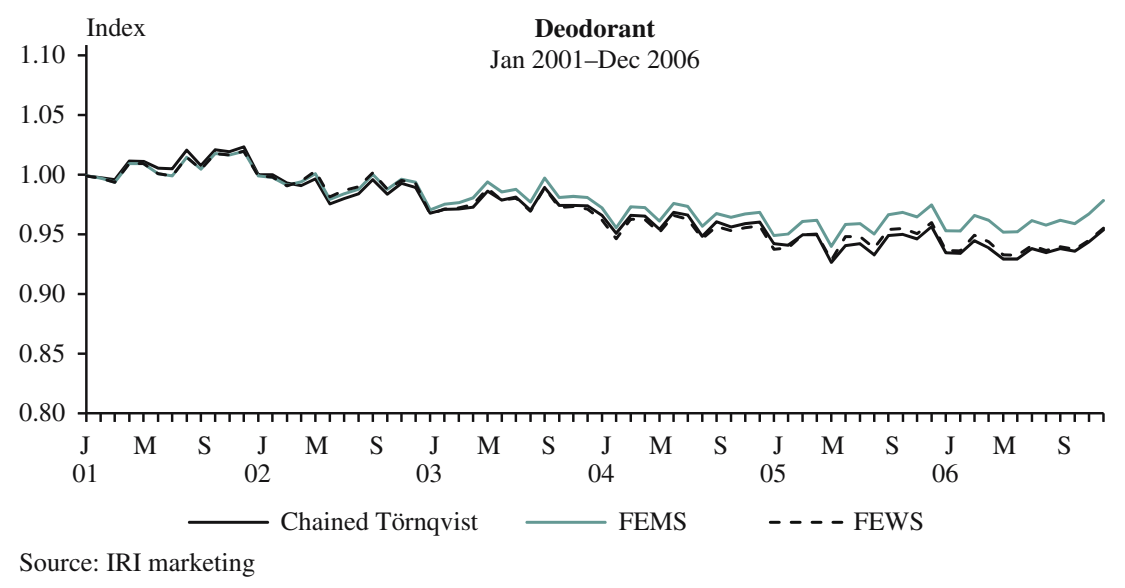

Fig. 9. Comparison of FEWS to other indexes for deodorant 


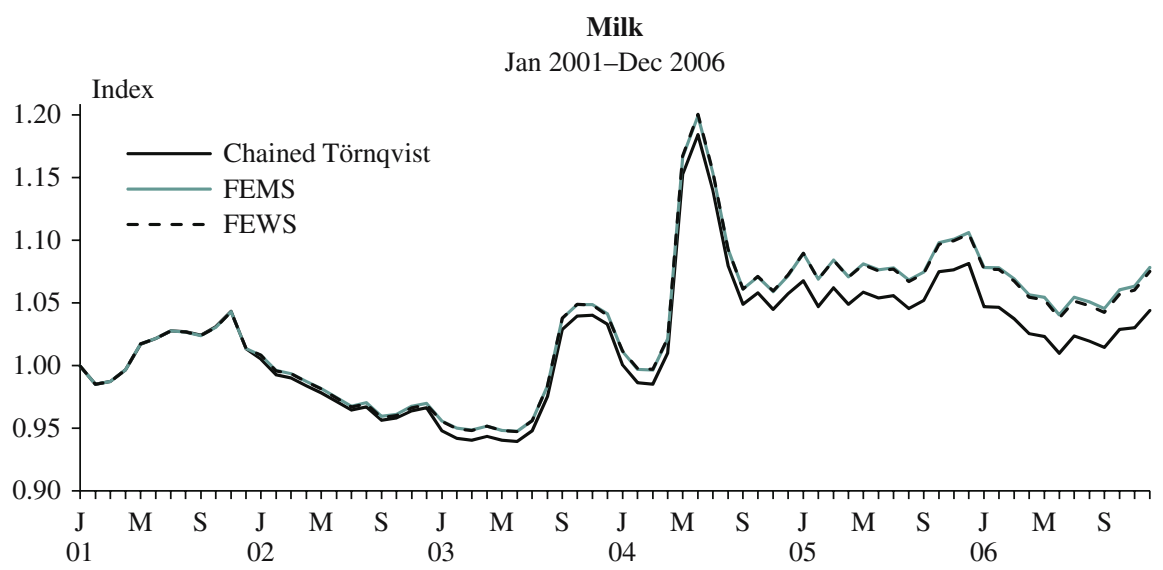

Source: IRI marketing

Fig. 10. Comparison of FEWS to other indexes for milk

are product categories for which the indexes do diverge, unlike most of the other product categories.

Table 3 gives the index values for the chained-Törnqvist, FEMS and FEWS indexes at the end of the six-year period analysed for all 30 products. Figures 13 and 14 present the same results graphically.

For many of the product categories, the three indexes track each other relatively closely. In general, there does not appear to be significant chain drift in the chained-Törnqvist index, in contrast to the findings in earlier research such as de Haan and van der Grient (2011) and Ivancic et al. (2011).

In Figure 9, for deodorant, the chained-Törnqvist and the FEWS index match closely, while the FEMS index tracks higher. An explanation for this could be that the valuing of characteristics by consumers is changing relatively quickly for this product category, and

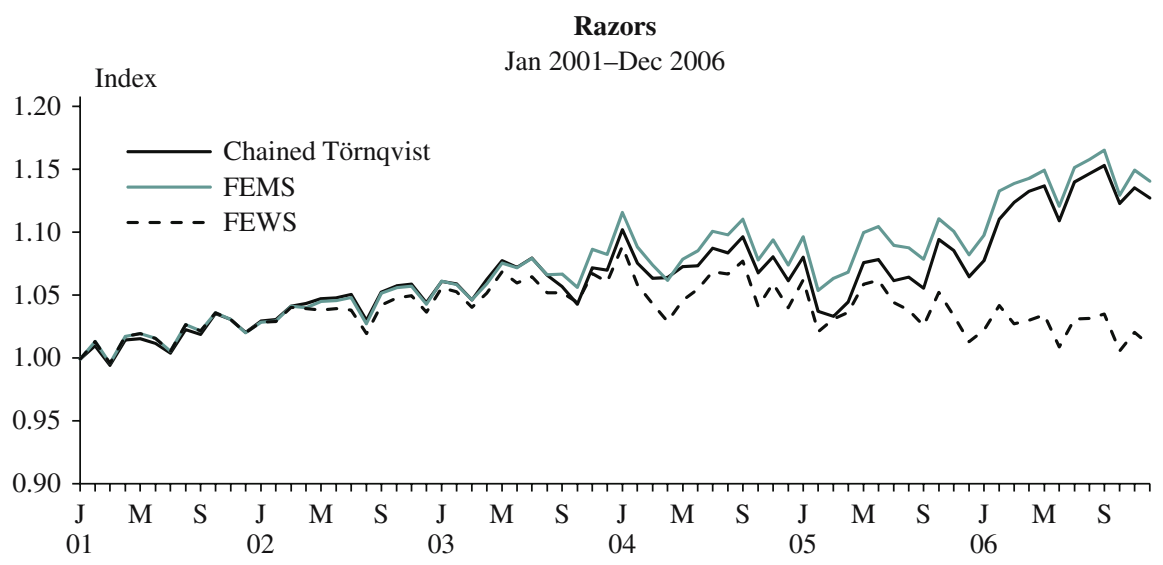

Source: IRI marketing

Fig. 11. Comparison of FEWS to other indexes for razors 


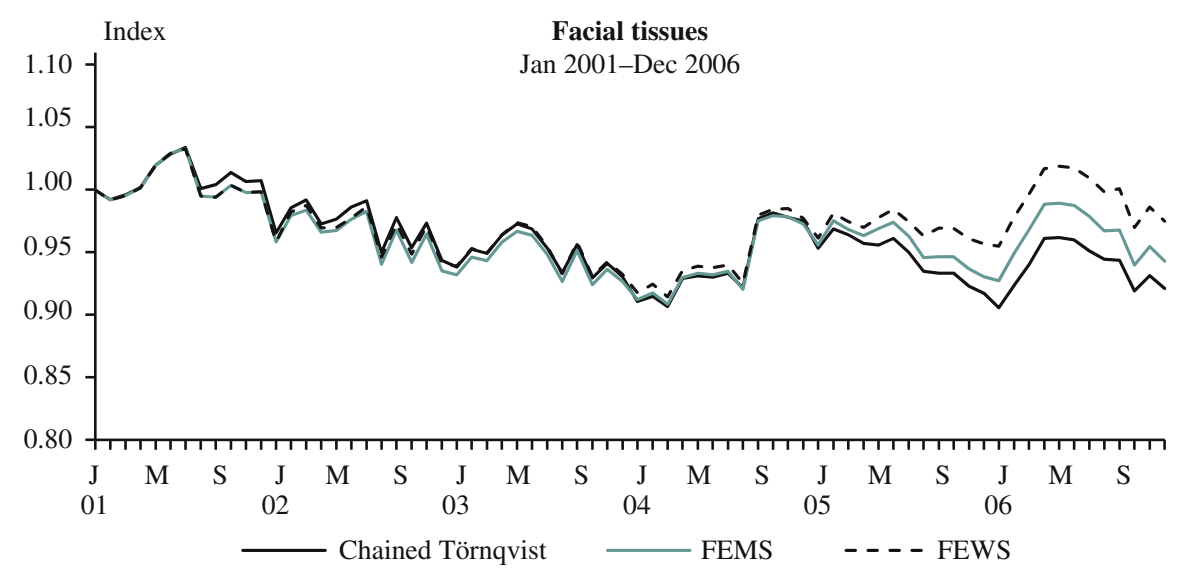

Source: IRI marketing

Fig. 12. Comparison of FEWS to other indexes for facial tissues

Table 3. Value of indexes at December 2006 (base =1 at January 2001)

\begin{tabular}{lccc}
\hline & Chained Törnqvist & FEMS & FEWS \\
\hline Beer & 1.098 & 1.095 & 1.085 \\
Blades & 1.159 & 1.160 & 1.144 \\
Carbonated beverages & 1.020 & 1.010 & 1.002 \\
Cigarettes & 1.194 & 1.184 & 1.190 \\
Coffee & 1.017 & 1.046 & 1.032 \\
Cold cereal & 1.059 & 1.056 & 1.020 \\
Deodorant & 0.955 & 0.979 & 0.956 \\
Diapers & 0.840 & 0.852 & 0.841 \\
Facial tissues & 0.921 & 0.943 & 0.975 \\
Frozen dinners and entrees & 0.981 & 0.984 & 0.972 \\
Frozen pizza & 0.948 & 0.958 & 0.938 \\
Household cleaner & 1.006 & 1.036 & 1.013 \\
Hotdogs & 1.170 & 1.171 & 1.180 \\
Laundry detergent & 0.938 & 0.967 & 0.949 \\
Margarine / butter blends & 1.121 & 1.121 & 1.111 \\
Mayonnaise & 1.114 & 1.109 & 1.089 \\
Milk & 1.044 & 1.079 & 1.076 \\
Mustard \& ketchup & 1.114 & 1.137 & 1.126 \\
Paper towels & 1.039 & 1.071 & 1.087 \\
Peanut butter & 1.023 & 1.047 & 1.035 \\
Photo supplies & 0.819 & 0.841 & 0.839 \\
Razors & 1.129 & 1.142 & 1.011 \\
Shampoo & 0.948 & 0.987 & 0.942 \\
Soup & 1.149 & 1.172 & 1.111 \\
Spaghetti / Italian sauce & 1.055 & 1.061 & 1.048 \\
Sugar substitutes & 1.088 & 1.086 & 1.090 \\
Toilet tissue & 1.106 & 1.093 & 1.101 \\
Toothbrushes & 0.911 & 0.931 & 0.904 \\
Toothpaste & 0.964 & 0.968 & 0.937 \\
Yoghurt & 1.016 & 1.029 & 1.026 \\
\hline
\end{tabular}




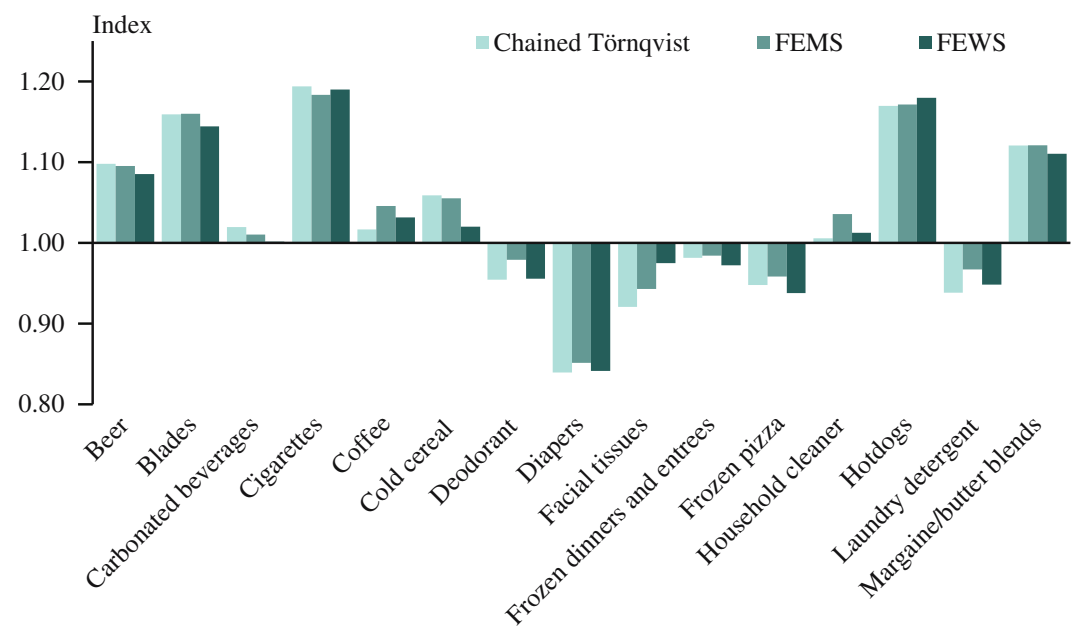

Source: IRI marketing

Fig. 13. Value of indexes at December 2006 (base $=1$ at January 2001)

so the implicit revision for the updating of the fixed-effects parameters of the FEWS is making a difference. The chained Törnqvist is based only on current and previous period data, so is 'up-to-date' in this sense. Any difference between the FEWS and the chained Törnqvist would be due to either

- chain drift in the chained-Törnqvist index, or

- the implicit price movements of new products entering and old products disappearing from the market differing from the price movements of matched products.

So, either these two factors are cancelling each other out for this product, or there is no chain drift in the Törnqvist and the implicit price movements from unmatched products are similar to those of matched products.

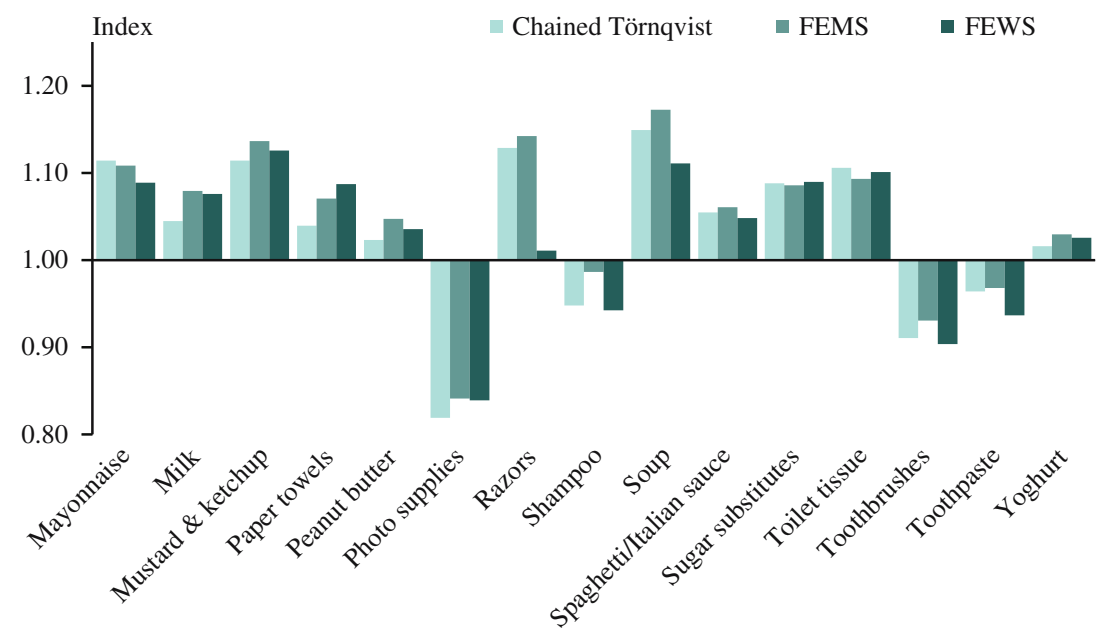

Source: IRI marketing

Fig. 14. Value of indexes at December 2006 (base $=1$ at January 2001) 
Figure 10 for milk shows the FEMS and FEWS indexes tracking virtually identically the indexes at December 2006 are 1.079 and 1.076 respectively - while there appears to be downwards chain drift influencing the chained-Törnqvist index.

Razors, shown in Figure 11, have the most unusual divergence in the three indexes of the 30 product categories analysed. From 2004 onwards the FEWS index trends downwards while the FEMS and chained-Törnqvist indexes continue to rise. This suggests that, during this period, there are downwards implicit price movements associated with the introduction of new products.

The three indexes for facial tissues, in Figure 12, all gradually drift apart. This is likely to suggest a combination of factors:

- downwards chain drift in the chained Törnqvist

- implicit price movements of new products differing from those of matched products and disappearing products (which the FEMS does reflect) and/or

- updating of fixed-effects parameters reflected by the FEWS but not the FEMS index.

Probably the main conclusion we can draw from the results for all 30 product categories, shown in Table 3 and Figures 13 and 14, is that there is no consistent pattern in the comparison of different index methods across all the products. This echoes the findings of previous research by Krsinich (2011a, 2013, 2014) and de Haan and Krsinich (2014). Further research is required to tease out the underlying factors driving different patterns of results, but this is not the focus of the present article.

\subsection{Online Data with no Quantities or Characteristics: Four New Zealand Consumer Electronics Products}

Statistics NZ was given access to 15 months of daily web-scraped online data from the Billion Prices Project at MIT for New Zealand consumer electronics products from one major New Zealand retailer.

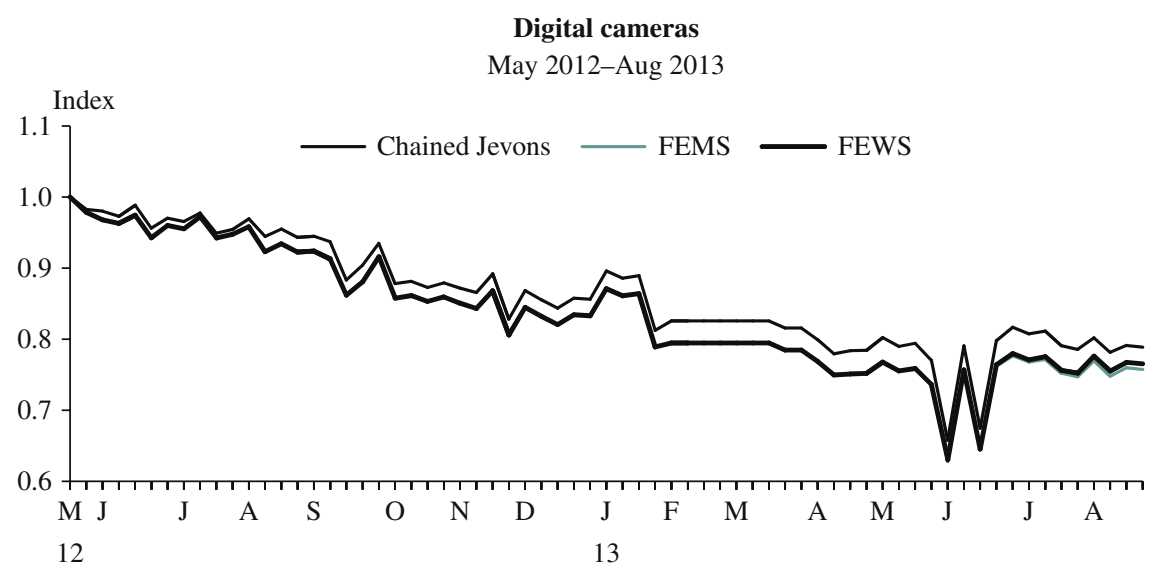

Source: Billion Prices Project @ MIT

Fig. 15. Comparison of FEWS to other indexes for digital cameras 


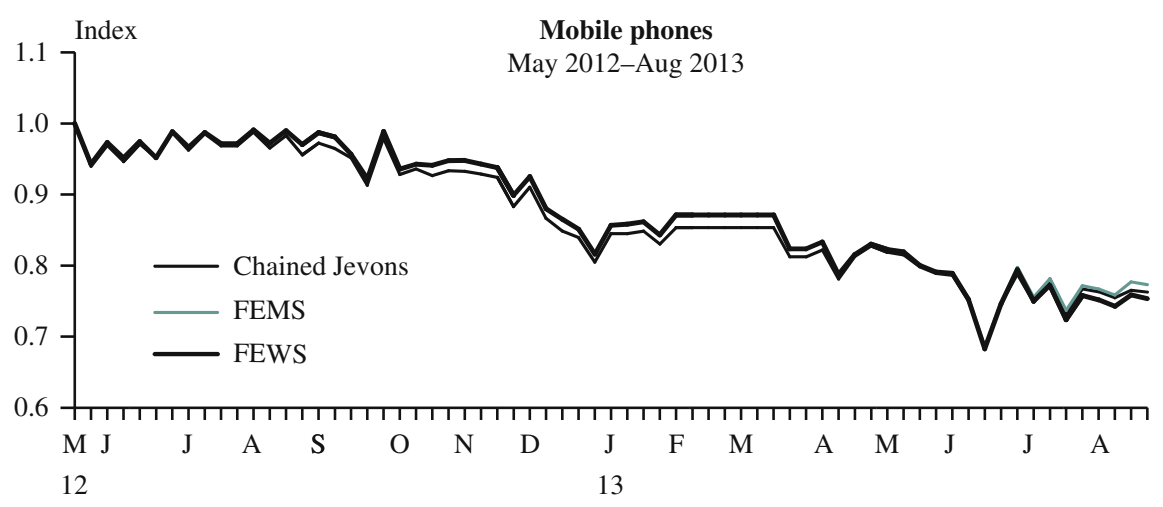

Source: Billion Prices Project @ MIT

Fig. 16. Comparison of FEWS to other indexes for mobile phones

Cavallo (2012) discussed possible approaches to quality adjusting this kind of online data, prompting this collaborative research into the fixed-effects index as a solution.

There are very few characteristics available in the online data but, as with scanner data, the products are identified by model name, and therefore any change in characteristics will generally correspond to a different product identifier. This means that the fixed-effects index can be used to implicitly quality adjust for all price-determining characteristics.

Another, less surmountable, limitation of online data is that quantities are not available. The unweighted index method used to estimate elementary-level price indexes by the Billion Prices Project and their commercial counterpart PriceStats is a daily-chained Jevons:

$$
P_{J}^{t-1, t}=\prod_{i \in M}\left(\frac{p_{i t}}{p_{i t-1}}\right)^{\frac{1}{m}}
$$

where $m$ is the number of matched products between periods $t-1$ and $t$.

An advantage of the chained-Jevons index is that it is transitive and therefore does not suffer from chain drift. However, unlike the FEWS index, the Jevons does not reflect the

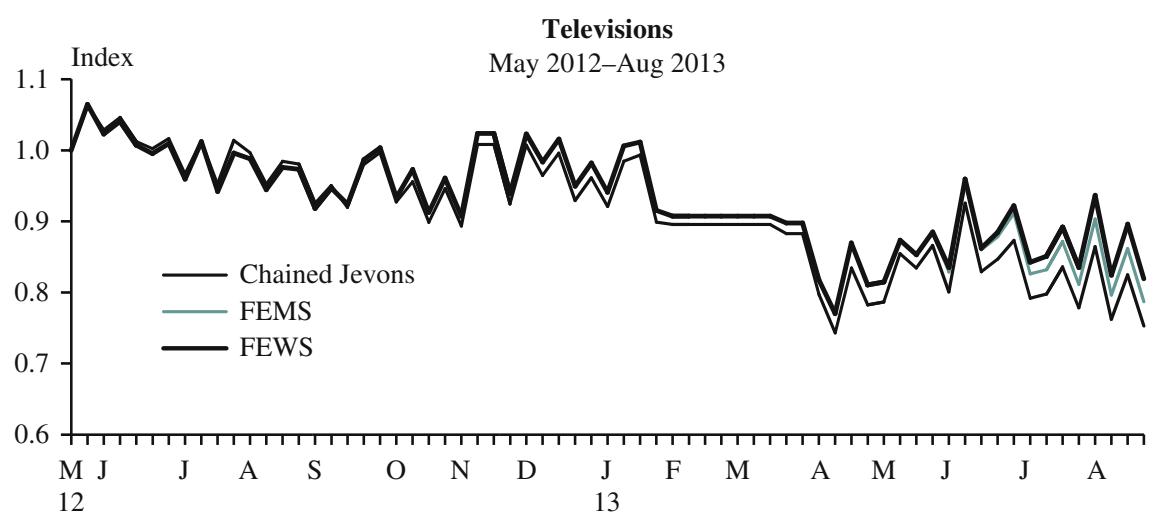

Source: Billion Prices Project @ MIT

Fig. 17. Comparison of FEWS to other indexes for televisions 
Table 4. Value of indexes at the end of August 2013 (base $=1$ at mid-May 2012)

\begin{tabular}{lccc}
\hline & Chained Jevons & FEMS & FEWS \\
\hline Mobile phones & 0.763 & 0.773 & 0.753 \\
Digital cameras & 0.788 & 0.757 & 0.765 \\
Televisions & 0.753 & 0.787 & 0.819 \\
\hline
\end{tabular}

implicit price movements of new and disappearing products at the time of their introduction and disappearance.

Krsinich (2015) shows that the noninclusion of quantities may not have as much of an impact as might be expected. The effect of having no quantities in online data is simulated by estimating FEWS indexes with and without expenditure share weights on GfK scanner data for consumer electronics.

For the analyses shown here, day-per-week (Saturday) samples of the daily data were used.

Figures 15 to 17 compare the (unweighted) FEWS index to the (unweighted) FEMS and the weekly chained-Jevons indexes. Note that the analysis period is only 15 months long, and the FEMS and the FEWS indexes will be equivalent for most of this period - that is, the first 53 of the full 72 weeks - as this is the length of the initial estimation window.

Note that there was a lapse in data collection between January and March 2013, which results in a flat index for each of the product categories over this period.

Table 4 gives the value of the indexes at the end of the 15 months.

Over the 15-month period, the three indexes are relatively similar. The difference between the chained-Jevons and the FEWS index for digital cameras and televisions reflects the impact of the implicit price movements of new and disappearing products which are reflected in the FEWS index, but not in the chained-Jevons index.

The high volatility of the indexes probably reflects the lack of smoothing by aggregation to monthly average prices, and the fact that the lack of quantities means that unusual price movements of low-selling products can have more of an impact on the index than they would in a weighted index. The coverage of the online data is also lower than that in the GfK scanner data, as it relates to just one, albeit large, New Zealand retailer.

\section{Conclusion}

This article has shown that the fixed-effects window-splice (FEWS) index produces nonrevisable quality-adjusted price indexes in the case of 'big data', such as scanner data or online data, where there is longitudinal price information at a detailed productspecification level.

There are two key factors behind this:

1. The fixed-effects index leverages off the longitudinal information in the data to implicitly quality adjust in a way that is shown to be equivalent to a fully interacted time-dummy hedonic index.

2. The use of a window splice implicitly revises so that the effects of new products are not systematically omitted as they would be with the more standard 'movement splice'. This is a form of implicit revision that also enables the estimation of fixed effects for all products to be updated. 
The FEWS index may be less appropriate for product areas where product characteristics or consumer preferences change rapidly, for example with seasonal variation or technological development. More research is required to understand and formulate the limits of the method's applicability in this context.

\section{Appendix 1}

\section{Derivation of Equation 6}

Restating and extending the working of de Haan and Hendriks (2013) so that all characteristics are treated as categorical and all interactions are included in the timedummy hedonic models along with the main effects, the predicted prices of item (i.e., product) $i$ in the base period 0 and the comparison periods $t$ are as follows.

$$
\begin{gathered}
\hat{p}_{i}^{0}=\exp \left(\hat{\delta}^{0}\right) \exp \left[\sum_{l=1}^{L} \hat{\beta}_{l} D_{i l}+\sum_{m=1}^{M} \hat{\beta}_{m} D_{i m}\right] \\
\hat{p}_{i}^{t}=\exp \left(\hat{\delta}^{0}\right) \exp \left(\hat{\delta}^{t}\right) \exp \left[\sum_{l=1}^{L} \hat{\beta}_{l} D_{i l}+\sum_{m=1}^{M} \hat{\beta}_{m} D_{i m}\right] ; \quad(\mathrm{t}=1, \ldots, \mathrm{T})
\end{gathered}
$$

Taking the geometric mean of the predicted prices for all items belonging to the samples $S^{0}$ and $S^{1}, \ldots, S^{T}$ gives the following.

$$
\begin{array}{r}
\prod_{i \in S^{0}}\left(\hat{p}_{i}^{0}\right)^{\frac{1}{N^{0}}}=\exp \left(\hat{\delta}^{0}\right) \exp \left[\left(\sum_{l=1}^{L} \hat{\beta}_{l} \sum_{i \in S^{0}} D_{i l}+\sum_{m=1}^{M} \hat{\beta}_{m} \sum_{i \in S^{0}} D_{i m}\right) / N^{0}\right] \\
\prod_{i \in S^{t}}\left(\hat{p}_{i}^{t}\right)^{\frac{1}{N^{t}}}=\exp \left(\hat{\delta}^{0}\right) \exp \left(\hat{\delta}^{t}\right) \exp \left[\left(\sum_{l=1}^{L} \hat{\beta}_{l} \sum_{i \in S^{t}} D_{i l}+\sum_{m=1}^{M} \hat{\beta}_{m} \sum_{i \in S^{t}} D_{i m}\right) / N^{t}\right]
\end{array}
$$

Dividing (20) by (19) and rearranging gives the following.

$$
\begin{aligned}
\exp \left(\hat{\delta}^{t}\right) & =\frac{\prod_{i \in S^{t}}\left(\hat{p}_{i}^{t}\right)^{\frac{1}{N^{t}}} \exp \left[\left(\sum_{l=1}^{L} \hat{\beta}_{l} \sum_{i \in S^{0}} D_{i l}+\sum_{m=1}^{M} \hat{\beta}_{m} \sum_{i \in S^{0}} D_{i m}\right) / N^{0}\right]}{\prod_{i \in S^{0}}\left(\hat{p}_{i}^{0}\right)^{\frac{1}{N^{0}}} \exp \left[\left(\sum_{l=1}^{L} \hat{\beta}_{l} \sum_{i \in S^{t}} D_{i l}+\sum_{m=1}^{M} \hat{\beta}_{m} \sum_{i \in S^{t}} D_{i m}\right) / N^{t}\right]} \\
& =\frac{\prod_{i \in S^{t}}\left(\hat{p}_{i}^{t}\right)^{\frac{1}{N^{t}}}}{\prod_{i \in S^{0}}\left(\hat{p}_{i}^{0}\right)^{\frac{1}{N^{0}}}} \exp \left[\sum_{l=1}^{L} \hat{\beta}_{l}\left(\bar{D}_{l}^{0}-\bar{D}_{l}^{t}\right)+\sum_{m=1}^{M} \hat{\beta}_{m}\left(\bar{D}_{m}^{0}-\bar{D}_{m}^{t}\right)\right]
\end{aligned}
$$

where $\bar{D}_{l}^{0}=\frac{\sum_{i \in S_{0}} D_{i l}}{N_{0}}$ and $\bar{D}_{l}^{t}=\frac{\sum_{i \in S_{t}} D_{i l}}{N_{t}}$ are the unweighted sample means of the dummy variable for category $l$ of the main effects and $\bar{D}_{m}^{0}=\frac{\sum_{i \in S_{0}} D_{i m}}{N_{0}}$ and $\bar{D}_{m}^{t}=\frac{\sum_{i \in S_{t}} D_{i m}}{N_{t}}$ are the 
unweighted sample means of the dummy variable for category $m$ of the second-order interactions.

Because the residual terms sum to zero in each period, (21) can be rewritten.

$$
\begin{aligned}
P_{T D(f u l l)}^{0 t} & =\exp \left(\hat{\delta}^{t}\right) \\
& =\frac{\prod_{i \in S^{t}}\left(p_{i}^{t}\right)^{\frac{1}{N^{t}}}}{\prod_{i \in S^{0}}\left(p_{i}^{0}\right)^{\frac{1}{N^{0}}}} \exp \left[\sum_{l=1}^{L} \hat{\beta}_{l}\left(\bar{D}_{l}^{0}-\bar{D}_{l}^{t}\right)+\sum_{m=1}^{M} \hat{\beta}_{m}\left(\bar{D}_{m}^{0}-\bar{D}_{m}^{t}\right)\right]
\end{aligned}
$$

And this is Equation (6) in the main text.

\section{Appendix 2}

\section{Intuition}

It is difficult to understand how it is possible for quality adjustment to happen without any explicit information on characteristics. The key point is that, in scanner and online data, products are defined at the detailed specification level - that is, at a level at which any change in price-determining characteristics would prompt a new identifier. Rather than estimating each characteristic's effect separately, the fixed-effects model is estimating the effect of each 'bundle' of characteristics corresponding to a particular product.

To get an intuition for how this works, first consider four products, all with the same set of characteristics, which are fixed across time. The standard approach to hedonic modelling fits the log of price against characteristics and time, so the graphs of logged price for each product can be visualised as shown below in Figure 18. $P^{\prime} O$ is the predicted logged price for the base product $P O$, and actual logged prices for each of the four products $P 0$ to $P 3$ differ from this predicted $P O$ each period by a normally distributed error term.

Now consider the situation where the products each have different characteristics from one another - that is, they are now four distinct products - and these characteristics are fixed across time at the product level - as they would be in the case of scanner data or online data, where barcodes or product names change if there is a change in characteristics.

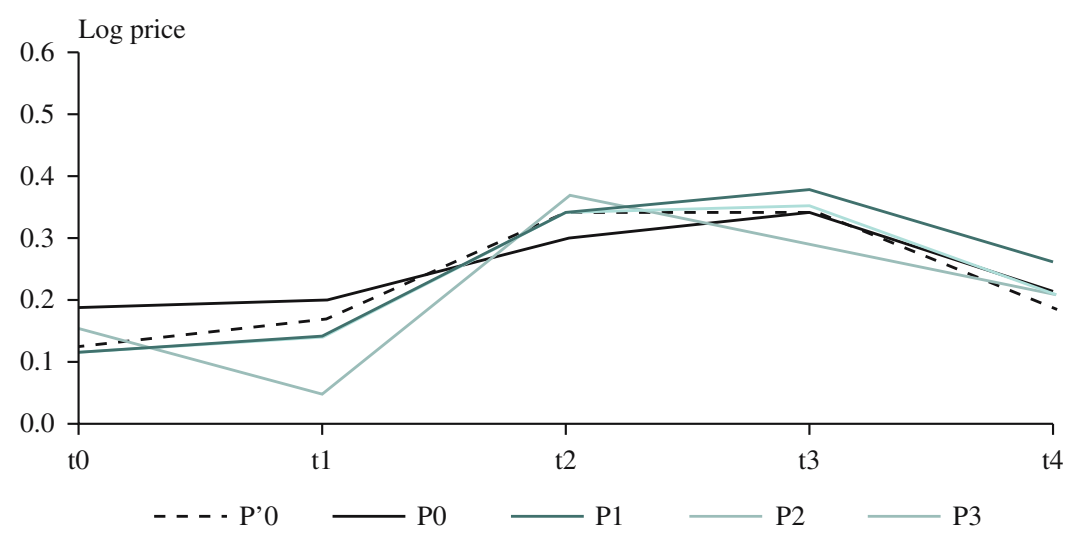

Fig. 18. All products with the same characteristics 
Assume that, within the estimation window of the hedonic model, characteristics' parameters are constant. Another, perhaps more useful, way of stating this is that the model will estimate the average value of each characteristic's parameter within the estimation window.

The net effect of each product's 'bundle of characteristics' is constant over time, so the logged price graph is moved up or down accordingly. This constant factor is the 'fixed effect' estimated by the fixed-effects model - that is, the product-specific intercept. This is shown below in Figure 19.

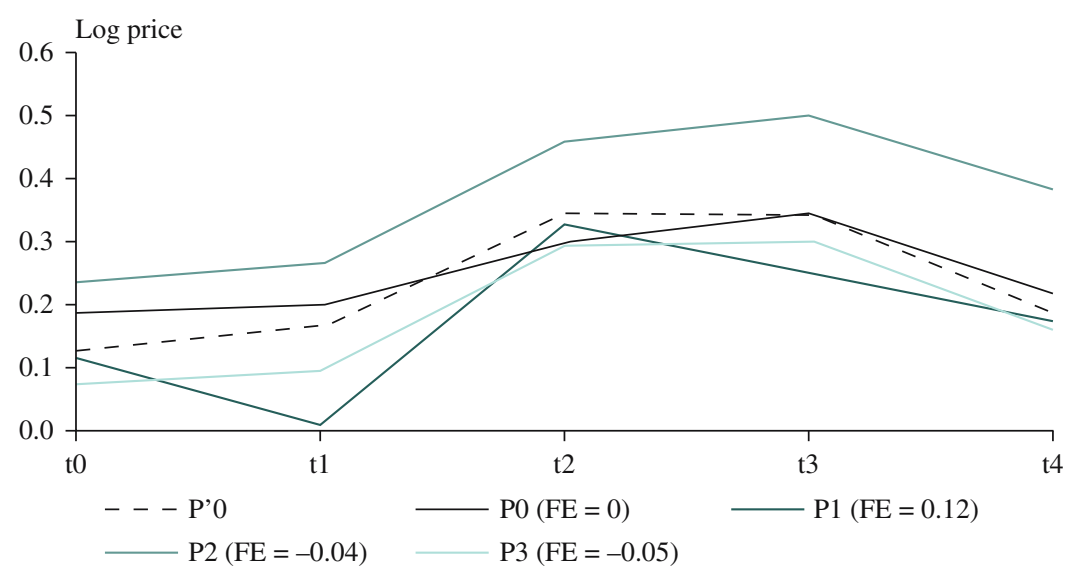

Fig. 19. Each product with a different set of characteristics

So, the fixed effects are estimated such that the sum of squares of the error terms is minimised for each of the products, resulting in the predicted log price for the base product $P^{\prime} O$ (i.e., the dashed line).

Consider, now, the situation of a new product $P 4$ entering at time $t 1$. Figure 20 shows the logged price of this new product $P 4$ over the full period from $t 0$ to $t 4$, alongside the logged prices of the other products.

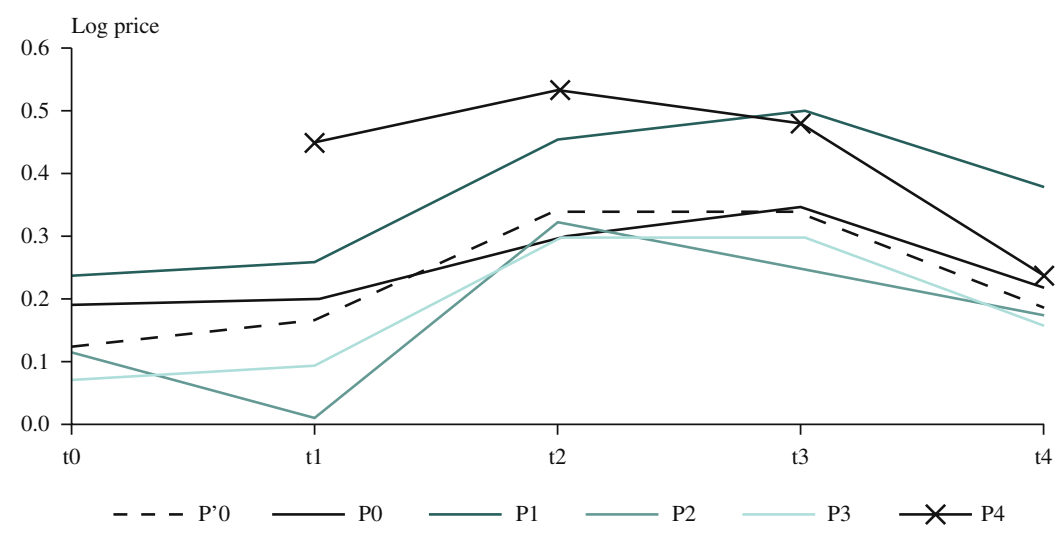

Fig. 20. New product entering at time 11 
In the period of introduction, the best estimate of the fixed effect of the new product $P 4$ - that which minimises the sum of squared error terms - is simply the distance between its $\log$ price and the predicted $\log$ price of the base item $P O$. This is shown below in Figure 21. Clearly, this is a trivial estimate, and the model (and therefore the index derived from it) is unaffected by the inclusion of the new product in this first period. Consequently, the corresponding implicit price movement associated with introducing this new product is simply the underlying price movement of the matched products.

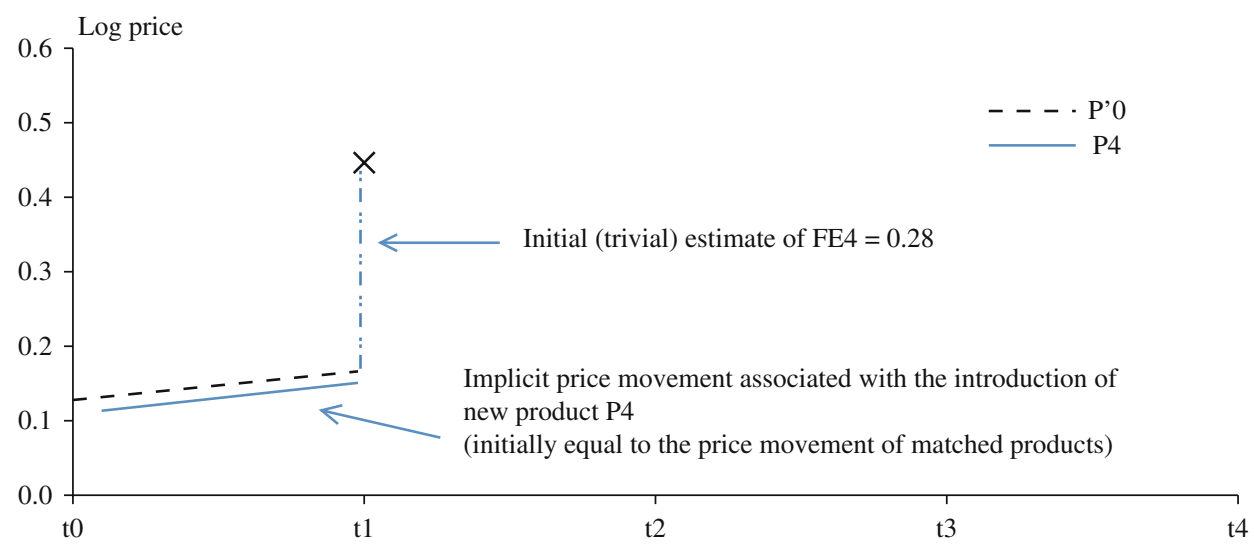

Fig. 21. Period of introduction of new product P5

This is the reason that, as noted by Diewert (2004), matched-model and fixed-effects indexes are equivalent when we consider only bilateral (two-period) model estimations.

The second price observation of $P 4$ enables non-trivial estimate of the fixed effect of new product $P 4$ (and consequently of the implicit price movement associated with its introduction), as shown in Figure 22. This can be visualised as shifting the logged price record of $P 4$ vertically up or down until the sum of squares of the error terms (indicated with bold lines) is minimised.

Note that for the purposes of this example it is assumed that the underlying predicted price movement of the base product $P^{\prime} O$ is unaffected by the new product $P 4$. Of course,

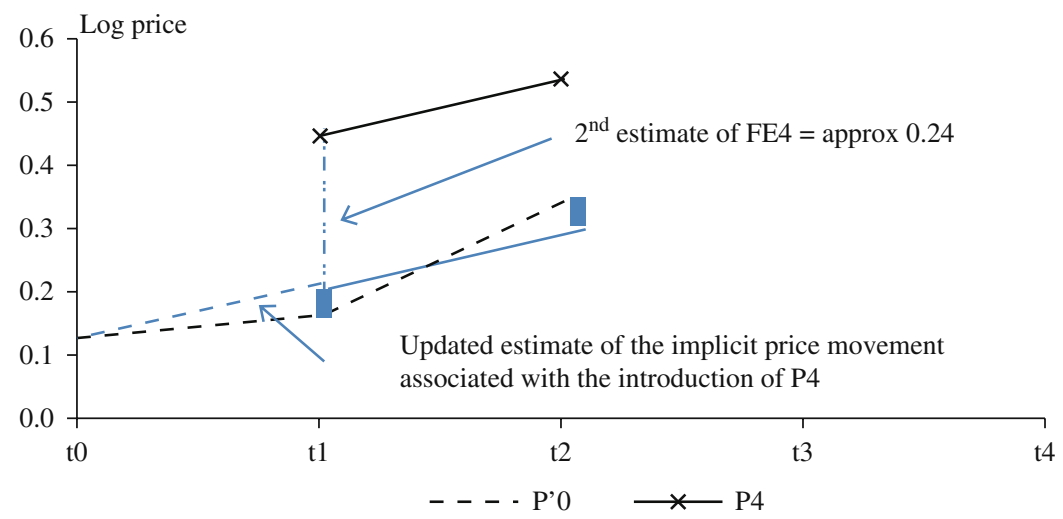

Fig. 22. Second price observation period for new product P5 
the predicted price will be slightly affected by the new product and it is the appropriate estimation of the new product's fixed effect, and therefore its influence on the resulting price index, that is the objective.

As more prices are observed for the new product $P 4$, the estimate of its fixed effect is updated accordingly. Figures 23 and 24 show how the estimate of the fixed effect gradually reduces each period as a result of fitting the longitudinal logged price record for this new product around the underlying logged price movement predicted from all products.

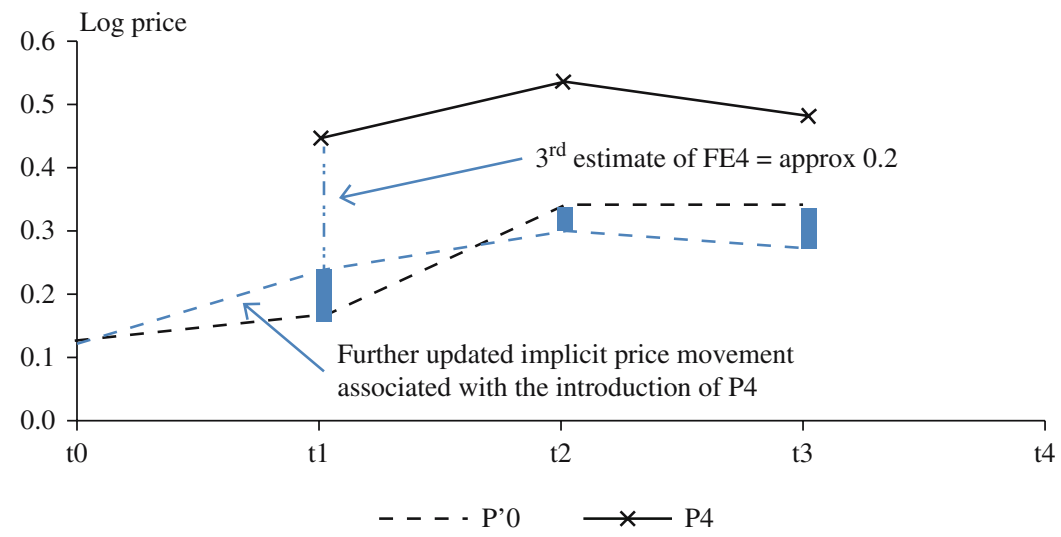

Fig. 23. Third price observation period for new product P5

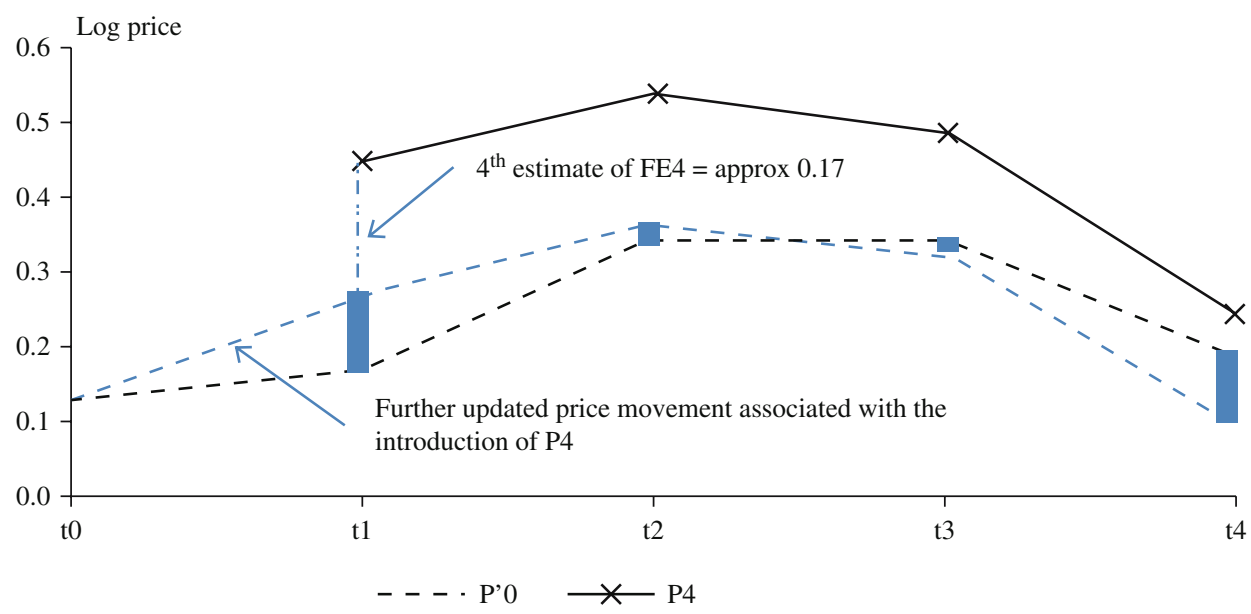

Fig. 24. Fourth price observation period for new product P5

Because the estimate of the fixed effect is trivial in the first period, at least two price observations are required before a new product can contribute meaningfully to the estimation. And with more price observations, the estimate of the fixed effect for the new product converges to its true value - that is, the estimate is being driven more by the shape of the longitudinal price record than by the error terms. The window splice described in Section 5 enables an implicit revision to be incorporated along with the most recent period's estimated movement to account for this updating of the fixed-effects estimates. 


\section{Appendix 3}

\section{Descriptive Statistics on the Three Data Sources}

Tables 5 to 7 show the average number of distinct products per month (for the GfK and IRI scanner data) and per week (for the BPP data, as represented by the Saturday sampling) for each product category.

They also show the percentage match rates after the first year for each product category, to indicate the rates of product turnover. These show that the turnover for consumer electronics can be very rapid - at its most extreme, only three percent and four percent of products are matched after the first year for laptop computers and desktop computers respectively. Even

Table 5. Average number of products per month, and match rate after the first year, for GfK consumer electronics scanner data

\begin{tabular}{lcr}
\hline & Number of products & Match rate $(\%)$ \\
\hline Camcorders & 88 & 41 \\
Desktop computers & 150 & 4 \\
Digital cameras & 289 & 23 \\
DVD players and recorders & 202 & 26 \\
Laptop computers & 432 & 3 \\
Microwaves & 152 & 33 \\
Portable media players & 161 & 18 \\
Televisions & 341 & 23 \\
\hline
\end{tabular}

Table 6. Average number of products per month, and match rate after the first year for IRI supermarket scanner data

\begin{tabular}{lrc}
\hline & Number of products & Match rate (\%) \\
\hline Beer & 2951 & 84 \\
Blades & 445 & 87 \\
Carbonated beverages & 4138 & 90 \\
Cigarettes & 1650 & 84 \\
Coffee & 2883 & 87 \\
Cold cereal & 1679 & 88 \\
Deodorant & 1039 & 83 \\
Diapers & 401 & 70 \\
Facial tissues & 234 & 80 \\
Frozen dinners and entrees & 1438 & 89 \\
Frozen pizza & 1247 & 93 \\
Household cleaner & 519 & 82 \\
Hotdogs & 929 & 93 \\
Laundry detergent & 785 & 81 \\
Margarine / spreads / butter blends & 258 & 94 \\
Mayonnaise & 358 & 90 \\
Milk & 2794 & 89 \\
Mustard and ketchup & 872 & 85 \\
Paper towels & 419 & 88 \\
Peanut butter & 251 & 92 \\
Photography supplies & 163 & 82 \\
Razors & 83 & 80 \\
Shampoo & 2216 & 76 \\
Soup & 1120 & 89 \\
Spaghetti / Italian sauce & 1418 & 91 \\
Sugar substitutes & 145 & 90 \\
Toilet tissue & 238 & 78 \\
Toothbrushes & 569 & 78 \\
Toothpaste & 731 & 75 \\
Yoghurt & 1751 & 95 \\
\hline & &
\end{tabular}


Table 7. Average number of products per week, and match rate after the first year for BPP consumer electronics online data

\begin{tabular}{lcc}
\hline & Number of products & Match rate (\%) \\
\hline Digital cameras & 94 & 49 \\
Mobile phones & 60 & 16 \\
Televisions & 51 & 18 \\
\hline
\end{tabular}

the consumer electronics product category with the highest match rate in the GfK scanner data - camcorders - has just $41 \%$ of products still being observed after the first year.

In contrast, the match rates for the supermarket products are much higher. These range from $70 \%$ for diapers to $95 \%$ for yoghurt.

\section{References}

Aizcorbe, A., C. Corrado, and M. Doms. 2003. "When Do Matched-Model and Hedonic Techniques Yield Similar Price Measures?” Working Paper no. 2003-14, Federal Reserve Bank of San Francisco. Available at: http://papers.ssrn.com/sol3/papers. cfm?abstract_id=550421 (accessed March 2016).

Bronnenberg, B.J., M. Kruger, and C.F. Mela. 2008. "The IRI Marketing Data Set" Marketing Science 27: 745-748. Available at: https://www.researchgate.net/ publication/259286152_The_IRI_marketing_data_set (accessed April, 2016).

Cavallo, A. 2012. “Overlapping Quality Adjustment Using Online Data.” Presentation to the 2012 Economic Measurement Group, Sydney, November 21-23, Australia. Available at: https://www.business.unsw.edu.au/research-site/centreforappliedeconomic research-site/Documents/A.\%20Cavallo\%20-\%20Overlapping\%20Quality\%20 Adjustment\%20Using\%20Online\%20Data.pdf (accessed April 2016).

de Haan, J. 2015a. "The Time-Product Dummy Method and Implicit Quality Adjustment." Unpublished draft, May 2015.

de Haan, J. 2015b. "Rolling Year Time Dummy Indexes and the Choice of Splicing Method." Paper presented at the $14^{\text {th }}$ meeting of the Ottawa Group, Tokyo. 20-22 May, 2015. Available at: http://www.stat.go.jp/english/info/meetings/og2015/pdf/t1s3room. pdf (accessed April 2016).

de Haan, J. and H. van der Grient. 2011. "Eliminating Chain Drift in Price Indexes Based on Scanner Data." Journal of Econometrics 161: 36-46. Doi: http://dx.doi.org/10.1016/ j.jeconom.2010.09.004.

de Haan, J. and R. Hendriks. 2013. "Online Data, Fixed Effects and the Construction of High-Frequency Price Indexes.” Paper presented at the 2013 Economic Measurement Group, Sydney, Australia. 28-29 November, 2013. Available at: https://www.business. unsw.edu.au/research-site/centreforappliedeconomicresearch-site/Documents/Jande-Haan-Online-Price-Indexes.pdf (accessed April 2016).

de Haan, J. and F. Krsinich. 2014. "Scanner Data and the Treatment of Quality Change in Non-Revisable Price Indexes.” Journal of Business \& Economic Statistics 32: 341-358. Doi: http://dx.doi/10.1080/07350015.2014.880059.

Diewert, W.E. 2004. "On the Stochastic Approach to Linking the Regions in the ICP." Discussion Paper no. 04-16, Department of Economics, University of British Columbia, 
Vancouver, Canada. Available at: http://papers.economics.ubc.ca/legacypapers/ dp0416.pdf (accessed March 2016).

Ivancic, L., W.E. Diewert, and K.J. Fox. 2011. "Scanner Data, Time Aggregation and the Construction of Price Indexes." Journal of Econometrics 161: 24-35. Doi: http://dx. doi.org/10.1016/j.jeconom.2010.09.003.

Krsinich, F. 2011a. "Price Indexes from Scanner Data: A Comparison of Different Methods." Paper presented at the $12^{\text {th }}$ meeting of the Ottawa Group, Wellington, New Zealand. 4-6 May, 2011. Available at: http://www.stats.govt.nz/ /media/Statistics/ottawa-group-2011/ Ottawa-2011-Papers/Krsinich-2011-paper-PImethods-comparison.pdf (accessed April 2016). Krsinich, F. 2011b. "Measuring the Price Movements of Used Cars and Residential Rents in the New Zealand Consumers Price Index." Paper presented at the $12^{\text {th }}$ meeting of the Ottawa Group, Wellington, New Zealand. 4-6 May, 2011. Available at: http://www. stats.govt.nz/ /media/Statistics/ottawa-group-2011/Ottawa-2011-Papers/Krsinich2011-paper-Rentals-cars.pdf (accessed April 2016).

Krsinich, F. 2013. "Using the Rolling Year Time-Product Dummy Method for Quality Adjustment in the Case of Unobserved Characteristics." Paper presented at the $13^{\text {th }}$ meeting of the Ottawa Group, Copenhagen, Denmark. 1-3 May, 2013. Available at: http://www.dst.dk/da/Sites/ottawa-group/ /media/Kontorer/12-Priser-og-forbrug/ Ottawa-Group/Frances\%20Krsinich\%202\%20Ottawa\%20Group\%202013\%20RYTPD \%20final.pdf (accessed April 2016).

Krsinich, F. 2014. "Fixed Effects with a Window Splice - Non-Revisable QualityAdjusted Price Indexes with No Characteristic Information." Paper presented at the meeting of the group of experts on consumer price indices, 26-28 May 2014, Geneva, Switzerland. Available at: http://www.unece.org/fileadmin/DAM/stats/documents/ece/ ces/ge.22/2014/New_Zealand_-_FEWS.pdf (accessed April 2016).

Krsinich, F. 2015. "Price Indexes from Online Data Using the Fixed-Effects WindowSplice Method." Paper presented at the $14^{\text {th }}$ meeting of the Ottawa Group, Tokyo, Japan. 20-22 May, 2015. Available at: http://www.stat.go.jp/english/info/meetings/og2015/ pdf/t1s2p7_pap.pdf (accessed April 2016).

Lamboray, C. and F. Krsinich. 2015. "A Modification of the GEKS Index When Product Turnover is High." Paper presented at the $14^{\text {th }}$ meeting of the Ottawa Group, Tokyo, Japan. 20-22 May, 2015. Available at: http://www.stat.go.jp/english/info/meetings/ og2015/pdf/t1s1p2_pap.pdf (accessed April 2016).

Melser, D. 2011. “Constructing Cost of Living Indexes Using Scanner Data.” Unpublished draft, September 2011 - an updated version of "Constructing High Frequency Indexes Using Scanner Data." Paper presented at the 12th meeting of the Ottawa Group, Wellington, New Zealand. 4-6 May, 2011. Available at: http://www.stats.govt.nz/ / media/Statistics/ottawa-group-2011/Ottawa-2011-Papers/Melser-2011-paper-Constructingindexes.pdf (accessed April 2016).

The Billion Prices Project. 2016. Available at: http://bpp.mit.edu (accessed April 2016).

Received August 2014

Revised October 2015

Accepted November 2015 